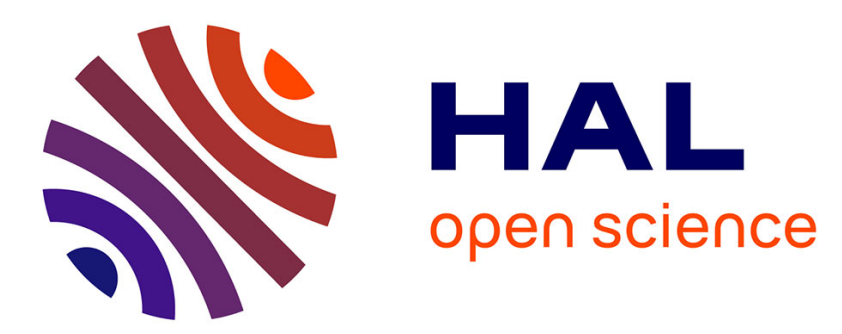

\title{
Evaluation of fatigue crack network growth in cast iron for different biaxial loading paths via full-field measurements
}

\author{
Zvonimir Tomicevic, Stéphane Roux, François Hild
}

\section{To cite this version:}

Zvonimir Tomicevic, Stéphane Roux, François Hild. Evaluation of fatigue crack network growth in cast iron for different biaxial loading paths via full-field measurements. International Journal of Fatigue, 2016, 92, pp.281-303. 10.1016/j.ijfatigue.2016.07.013 . hal-01383909

\section{HAL Id: hal-01383909 \\ https://hal.science/hal-01383909}

Submitted on 19 Oct 2016

HAL is a multi-disciplinary open access archive for the deposit and dissemination of scientific research documents, whether they are published or not. The documents may come from teaching and research institutions in France or abroad, or from public or private research centers.
L'archive ouverte pluridisciplinaire HAL, est destinée au dépôt et à la diffusion de documents scientifiques de niveau recherche, publiés ou non, émanant des établissements d'enseignement et de recherche français ou étrangers, des laboratoires publics ou privés. 


\title{
Evaluation of fatigue crack network growth in cast iron for different biaxial loading paths via full-field measurements
}

\author{
Zvonimir Tomičevića ${ }^{a, b}$, Stéphane Roux ${ }^{\mathrm{b}}$, François Hild ${ }^{\mathrm{b}}$ \\ ${ }^{a}$ Department of Engineering Mechanics, Faculty of Mechanical Engineering and Naval \\ Architecture, University of Zagreb, Ivana Lučića 5, 10000 Zagreb, Croatia \\ ${ }^{b}$ LMT-Cachan, ENS Cachan/CNRS/University Paris-Saclay \\ 61 avenue du Président Wilson, 94235 Cachan Cedex, France
}

\begin{abstract}
This paper proposes a new method for monitoring fatigue crack network initiation and growth based on Digital Image Correlation (DIC), and reports on in-plane biaxial fatigue experiments with two different loading paths and two amplitudes on nodular graphite cast iron. The central thinned part of cross-shaped samples is observed on two scales. Regularized Digital Image Correlation (DIC) is used to measure displacement fields and to reveal DIC residuals, i.e., image differences that cannot be accounted for with registration. The detailed strain histories are compared at different scales and for the different loading regimes. DIC and mechanical residuals, i.e., local deviations of the displacement field from being the solution to an elastic problem, enable for the crack network detection and quantification with respect to the number of cycles. The relative damage severity and fatigue lifetime of different loading paths experiencing identical load magnitudes are analyzed for the tested material.
\end{abstract}


Keywords: Biaxial experiments; Crack network; Digital Image Correlation; Regularization; Residuals.

\section{Introduction}

The prediction of fatigue life of structures is still a difficult task because it is a multiscale process. For defect-free media, the major part of damage growth corresponds to multiple initiations and propagations of short cracks, which eventually coalesce to form a macroscopic crack. To account for such phenomena, different models have been proposed [20, 23, 17, 2, 13, 36, 21, 26]. However, on the experimental side, the quantitative analysis of such phenomena remains a difficult and challenging task.

In this paper it is proposed to utilize digital image correlation (DIC [29]) to study the development of fatigue crack networks. DIC has been used to detect and quantify fatigue crack growth $[30,15,19,9,34,10,22,18]$. All the cited references essentially deal with a single crack. Only few studies have reported results on fatigue crack networks [12, 25, 16]. In the present work, it is proposed to use an optical setup enabling for two scale observations in biaxial tests. Such configurations have already been utilized to analyze macroscopic cracks $[1,35,6]$. However, they all used an artificial random pattern at both scales of observation, with the risk of masking the occurrence of microcracks. For this reason, the bare sample surface was used herein.

This study focuses on the fatigue behavior of spheroidal graphite (SG) cast iron under in-plane biaxial experiments. The low ductility of the mate- 
rial [33] induces the formation of crack networks during cyclic loading. The strain fields and correlation residuals are analyzed to detect multiple crack initiations via DIC simultaneously performed on both scales. Due to the ability of multiaxial experiments to prescribe a wide variety of loading histories two different paths, namely, "equibiaxial" (simultaneous loading and unloading in the two arm directions) and "square" (i.e., successive loading and unloading in the two arm directions as detailed below) will be used. One of the challenging parts of this work is the performance of DIC related to the poor natural texture of the studied material. A regularization technique [31] is used to overcome this limitation. From the measured displacement fields, the strain histories are extracted and compared on both scales. For each loading path two different tests are considered. The first one is designed to initiate the crack network during the first cycle. The second one is set so that a first stage of cycling takes place without cracks. Correlation and mechanical residuals obtained via DIC analyses are presented in order to reveal the crack network history.

\section{Experimental protocol}

\subsection{In-plane biaxial experiments}

The in-plane biaxial experiments presented herein are carried out on the triaxial servo-hydraulic machine Astrée [27, 7, 8, 11, 24, 10, 14, 32]. For the present experiments, four horizontal actuators are used. The material studied herein is an SG cast iron whose chemical composition is reported in 
Table 1.

Table 1: Chemical composition in vol. \% of the studied grade of SG cast iron

\begin{tabular}{|c|c|c|c|c|c|c|c|c|c|c|}
\hline $\mathrm{C}$ & $\mathrm{Si}$ & $\mathrm{Mn}$ & $\mathrm{P}$ & $\mathrm{S}$ & $\mathrm{Cr}$ & $\mathrm{Ni}$ & $\mathrm{Mo}$ & $\mathrm{Cu}$ & $\mathrm{Mg}$ & $\mathrm{Fe}$ \\
\hline 2.26 & 2.09 & 0.15 & 0.041 & $<0.01$ & 0.04 & 0.63 & $<0.01$ & 0.025 & 0.06 & bal. \\
\hline
\end{tabular}

The main feature of SG cast iron is its heterogeneous microstructure (Figure 1). SG cast iron consists of a ferrite/pearlite matrix containing randomly distributed spheroidal graphite inclusions (Figure 1(a)). Due to the size of the biaxial samples it was necessary to cast a bigger block with dimensions $280 \times 280 \times 500 \mathrm{~mm}^{3}$. The heat-treatment and especially the cooling rate of the cast part had to be adapted to such sizes. Consequently the nodules are not spherical (i.e., the mean circular shape factor (CSF) is equal to 0.62 , and the nodularity by area when the $\mathrm{CSF}>0.5$ is equal to $70 \%$ ). By revealing the secondary microstructure (Figure 1(b)) it is possible to observe the volume fraction of graphite nodules $(10 \%)$, ferrite $(31 \%)$ and pearlite $(59 \%)$ grains. 


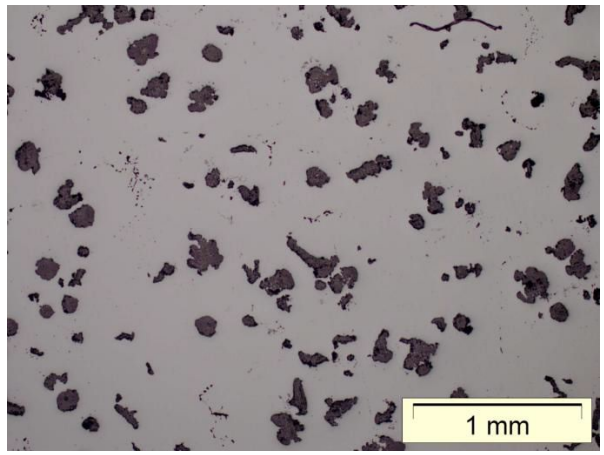

(a) Polished primary microstructure

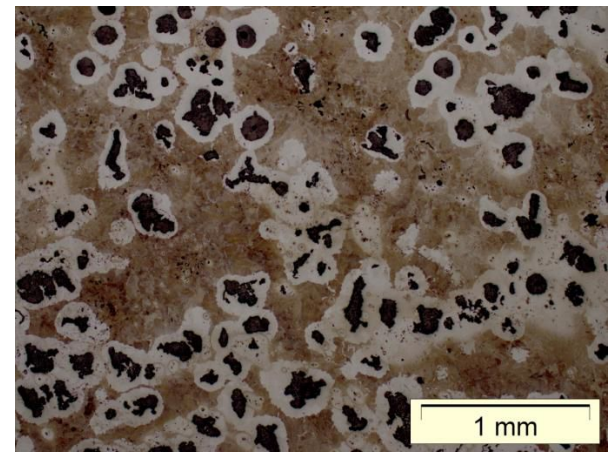

(b) Etched secondary microstructure with nitric acid

Figure 1: Metallography of the studied SG cast iron

In spite of this heterogeneous microstructure, at the macroscopic level the mechanical behavior of SG cast iron can be considered as homogeneous with isotropic properties. From previous work carried on the same material (i.e., quasi-static uniaxial tests) low elongation to failure was reported (i.e., $4.85 \%$ ) with a yield stress of $290 \mathrm{MPa}$, and an ultimate tensile strength of $460 \mathrm{MPa}$ [33]. This phenomenon is linked to the presence of pearlite in rather high volume fraction and very dense distribution of irregular nodules.

The in-plane biaxial test consists in loading a cross-shaped specimen in tension and/or compression along two perpendicular directions. The samples considered herein have a maltese cross shape that is thinned in the central part to form spherical caps. This uneven thickness gives rise to higher stress levels in the central region, and this stress enhancement is designed to induce crack initiation within this zone (Figure 2). 


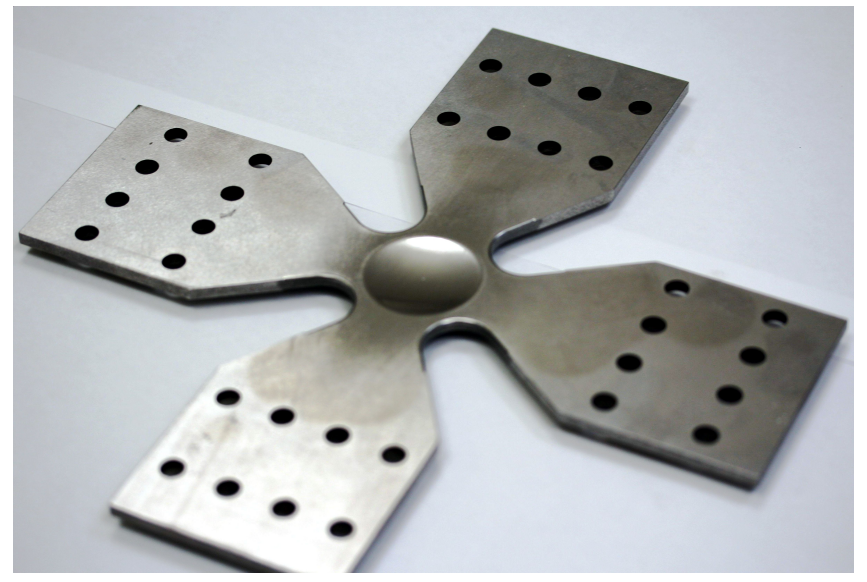

Figure 2: Maltese cross-shaped specimen designed for in-plane biaxial experiments

The global size of the sample is $274 \times 274 \mathrm{~mm}^{2}$. The lowest thickness at the center is equal to $1 \mathrm{~mm}$ (i.e., ten times the average graphite particle size) and gradually increases to $5 \mathrm{~mm}$ out of the gauge zone, which creates a calotte with of circular base $30 \mathrm{~mm}$ in diameter. The dimension of the fillets of the gripping arms is $12 \mathrm{~mm}$.

\subsection{Two-scale optical setup}

Since the geometry of the specimen is complex, the natural choice is to use full-field measurements to have access to the displacement/strain fields during the experiment. The goal of the experiments presented herein is to observe the material response to biaxial loading regimes on two scales. In order to capture displacement fluctuations a two-scale optical setup is selected (Figure 3). The thinned central part of the cross-shaped specimen was observed on both sides (Figure 3(a-b)). The bottom side of the sample 
is observed on a macroscale (Figure 3(b)) for which the camera monitors the whole gauge zone. Images were taken with a CCD camera $\left(\right.$ Dalsa $^{\mathrm{TM}}, 12$-bit digitization) and a telecentric lens with $\times 4$ magnification (Figure $3(\mathrm{c})$ ). The picture definition is $1024 \times 1024$ pixels with a physical pixel size of $48 \mu \mathrm{m}$ (Figure 3(d)). This optical setup is classical for DIC analyses.

For optimal DIC conditions, the sample surface is illuminated with a perpendicular light and the camera sensor is parallel with the observed zone. To achieve a diffuse illumination, oil paper is placed on the beam-splitter surface illuminated by a DedoCool ${ }^{\mathrm{TM}}$ lamp. Another CCD camera (PixelFly ${ }^{\mathrm{TM}}, 12-$ bit digitization, see Figure $3(\mathrm{f})$ ) equipped with $\times 1$ magnification telecentric lens is used to monitor the gauge zone. Mesoscale pictures (Figure 3(g)) have a definition of $1280 \times 1024$ pixels with a physical pixel size of $6.7 \mu \mathrm{m}$. The gauge zone has a size of $8.6 \times 6.9 \mathrm{~mm}$. 
(a)

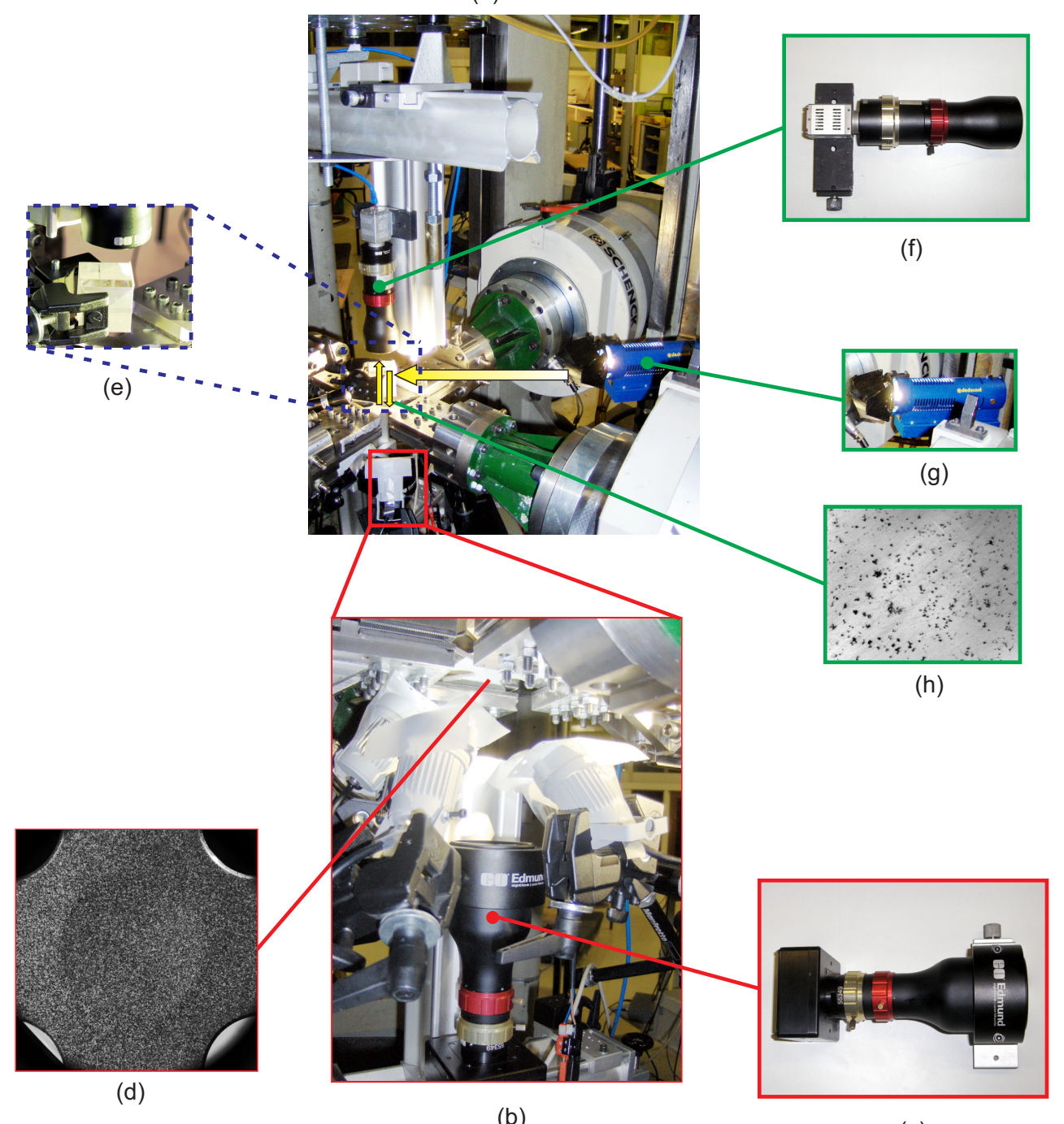

(b)

(c)

Figure 3: Two-scale experimental setup. (a) Mesoscale optical setup, (b) macroscale optical setup, (c) CCD camera Dalsa ${ }^{\mathrm{TM}}$ with telecentric lens ( $\times 4$ magnification), (d) macroscale image, (e) beam-splitter, (f) CCD camera PixelFly ${ }^{\mathrm{TM}}$ with telecentric lens ( $\times 1$ magnification), (g) DedoCool ${ }^{\mathrm{TM}}$ light source, (h) mesoscale image

Before conducting the two-scale analyses, the surfaces of the investigated 
regions of interest (ROIs) are prepared. To enable for DIC analyses of macroscale pictures an artificial pattern is applied. A fine speckle pattern is printed with an airbrush (i.e., ink jet printer, see Figure 4(a)). From the two types of microstructure presented in Figure 1 the primary one is found to be more suitable for DIC measurements. Hence, on the opposite side of the sample the gauge zone is polished (Figure 4(b)) in order to reveal the microstructure of SG cast iron. At the mesoscale, the graphite nodules are used as a natural pattern, which is very difficult to analyze with conventional DIC procedures [31] (see Figure 3(h)). However, a regularized DIC technique (see Section 2.3) revealed suitable for providing a satisfactory displacement uncertainty (see Appendices A and B).

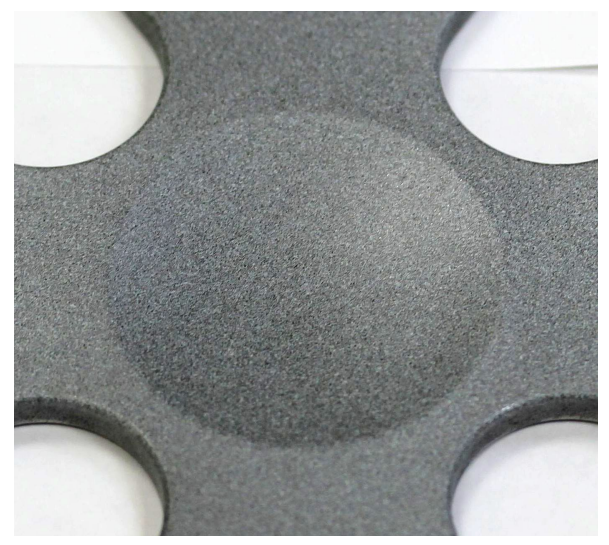

(a)

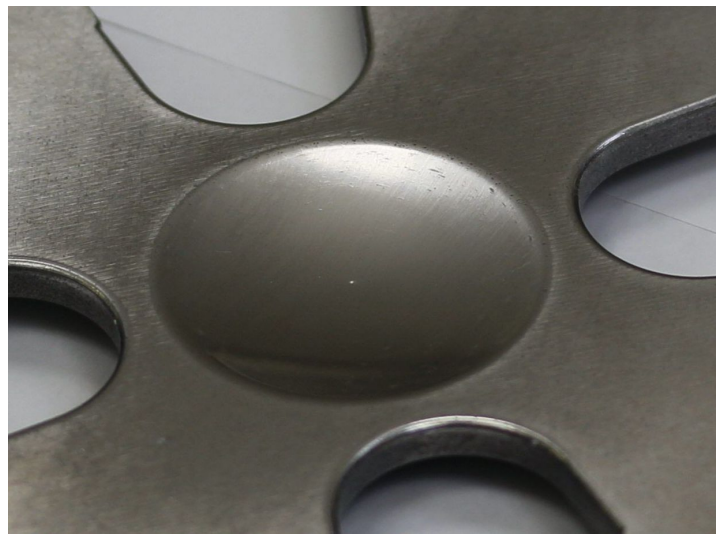

(b)

Figure 4: The two prepared sides of the cross-shaped specimen for two-scale observations.

(a) Artificial texture for macro- and (b) polished surface for mesoscale observations 


\subsection{Biaxial loading cases}

A first series of experiment is performed by applying equibiaxial loadings. The loading history (Figure 5(a)) corresponds to a proportional in-plane biaxial loading where both perpendicular axes are loaded and unloaded at the same level simultaneously. Conversely, in the so-called square regime (Figure 5(b)), the specimen is sequentially loaded and unloaded one direction at a time, so that in the $\left(F_{1}, F_{2}\right)$ plane, the loading path follows a square, whose diagonal is precisely the equibiaxial loading.

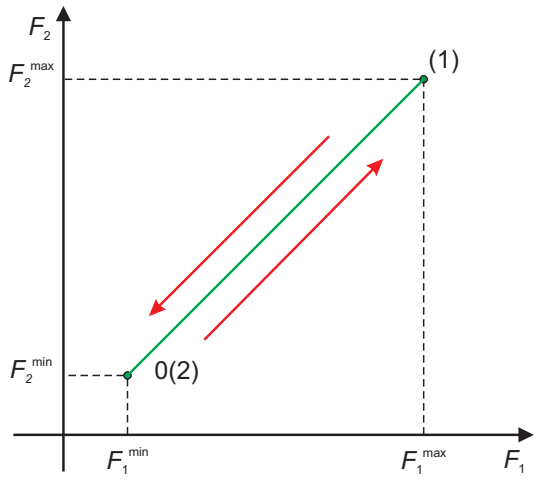

(a) Equibiaxial loading path

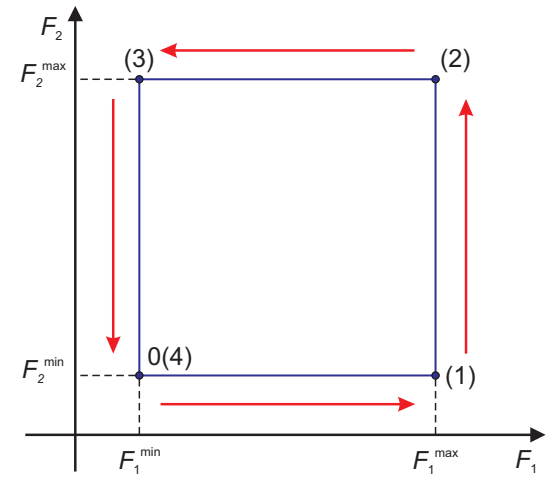

(b) Square loading path

Figure 5: One cycle for (a) equibiaxial and (b) square loading histories. Equibiaxial loading consists of two (i.e., (1)-(2)) characteristic points while square history has four (i.e., (1)-(2)-(3)-(4))

The tests are carried out at a frequency of $10 \mathrm{~Hz}$. The first series of twoscale fatigue experiments (see Figure 3 ) is conducted in an equibiaxial loading regime. Two different maximum load levels (see Table 2) are prescribed to investigate the lifetime and crack network formation during cycling. The tests 
are detailed in Section 3. A crack network can be detected at the first cycle (i.e., EBE-1 experiment where the maximum applied force $F^{\max }=40 \mathrm{kN}$ ). Lower levels are prescribed in a second experiment $\left(F^{\max }=20 \mathrm{kN}\right.$ and $25 \mathrm{kN}$ ), where microcrack initiation requires some cycles. Crack detection in this case is more complex and challenging. This case is labeled EBE-2 test.

Table 2: Definition of the equibiaxial and square loading paths, respectively referred to with the acronyms EBE and SBE

\begin{tabular}{|c|c|c|c|c|c|c|}
\hline & $\begin{array}{l}F_{1}^{\max } \\
(\mathrm{kN})\end{array}$ & $\begin{array}{l}F_{2}^{\max } \\
(\mathrm{kN})\end{array}$ & $\begin{array}{l}F_{2}^{\min } \\
(\mathrm{kN})\end{array}$ & $\begin{array}{l}F_{1}^{\min } \\
(\mathrm{kN})\end{array}$ & $\begin{array}{l}N_{\text {failure }} \\
\text { (cycles) }\end{array}$ & $\begin{array}{c}\sigma_{\max }^{v M} \\
(\mathrm{MPa})\end{array}$ \\
\hline EBE-1 & 40 & 40 & 0.1 & 0.1 & 18,224 & 395 \\
\hline \multirow{2}{*}{ EBE-2 } & 20 & 20 & 0.1 & 0.1 & $1,000,000$ & 240 \\
\hline & 25 & 25 & 0.1 & 0.1 & 550,354 & 280 \\
\hline SBE-1 & 40 & 40 & 0.1 & 0.1 & 163 & 505 \\
\hline SBE-2 & 20 & 20 & 0.1 & 0.1 & 247,065 & 295 \\
\hline
\end{tabular}

FE simulations were performed in order to assess the von Mises stress levels (i.e., $\sigma_{\max }^{v M}$ ) at the pole of the spherical cap for the two equibiaxial loading regimes (Table 2). Isotropic elasticity, J2-flow rule and nonlinear (i.e., Armstrong-Frederick) kinematic hardening were used to model the material behavior [32]. The computed equivalent von Mises stress for the maximum load level of EBE-1 test is equal to $395 \mathrm{MPa}$. This level lies between the yield stress and ultimate tensile strength. Conversely, the first part of EBE-2 test leads to a maximum stress $\sigma_{\max }^{v M}=240 \mathrm{MPa}$, which is $50 \mathrm{MPa}$ 
lower than the macroscopic yield stress. The second part of the test induces a maximum value $\sigma_{\max }^{v M}=280 \mathrm{MPa}$ that is closer to macroscopic yield stress (i.e., $290 \mathrm{MPa})$.

The second series of fatigue experiments considers two square loading paths. For comparison purposes the loading history of the square regime was chosen with the same load levels (Table 2). The square fatigue experiments are performed in a fully load-controlled mode. From the acquired images the mean strain levels are estimated with a DIC gauge (see Section 2.3). Two different cases (as for the equibiaxial loading regime) are considered to study microcrack initiation. The SBE-1 test is chosen because microcracks are detected at the first loading cycle while cracks initiate at a later stage for the SBE-2 test. The estimated stress levels via FE simulations are reported in Table 2. Even though the maximum load levels are identical, the maximum equivalent stresses are higher for the square history. The estimated equivalent stress level for test SBE-1 is equal to $505 \mathrm{MPa}$, which is greater than the ultimate tensile strength. It is expected that more microcracks will be initiated for this loading regime. This result also explains why only 163 cycles could be applied prior to failure. For the lowest load level (i.e., SBE-

2 test) the maximum equivalent stress is approximately the same as the macroscopic yield stress.

\subsection{Digital Image Correlation}

DIC is a widely used full-field measurement technique [29]. It is based 
on the conservation of gray levels between a reference image $f(\mathbf{x})$ and a deformed one $g(\mathbf{x})$. A global approach [5, 28, 3], which is applied herein, is an initial framework for mechanics-aided DIC. The sought displacement field is written in a general discretization scheme as $\mathbf{u}(\mathbf{x})=\sum_{n} u_{n} \boldsymbol{\psi}_{n}(\mathbf{x})$, where $\boldsymbol{\psi}_{n}$ are chosen fields. After integration over the whole ROI, the global "correlation residual" $\Phi_{c}$ is defined as

$$
\Phi_{c}^{2}=\sum_{\mathrm{ROI}}|f(\mathbf{x})-g(\mathbf{x}+[\boldsymbol{\psi}(\mathbf{x})]\{\mathbf{u}\})|^{2}
$$

In the following, correlation residual fields will be analyzed to highlight the unresolved differences between images of the current and reference states. To make the correlation residual field dimensionless, a normalized value $\hat{\Phi}_{c}$ is used, which corresponds to the root mean square average of $\varphi_{c}(\mathbf{x})=|f(\mathbf{x})-g(\mathbf{x}+[\boldsymbol{\psi}(\mathbf{x})]\{\mathbf{u}\})|$ divided by the dynamic range of gray levels $\left(\max _{\mathbf{x} \in R O I}[f(\mathbf{x})]-\min _{\mathbf{x} \in R O I}[f(\mathbf{x})]\right)$. After DIC analyses, all differences that can be explained by the specific kinematics of the surface are erased. The remaining differences correspond to local violations of the gray level conservation, or violation of the assumed smoothness of the displacement field (i.e., here continuity). Therefore, the correlation residual is the ideal field in which micro-cracks are expected to pop up. The following study will show that this is a very powerful tool whose potential is often ignored in DIC studies. Moreover, the spatial definition of the correlation residual field is the original pixel size. 


\subsubsection{Regularized DIC}

Regularized DIC (R-DIC) enforces mechanical admissibility in an FE sense with the equilibrium gap [8]. If linear elasticity were applicable, $[\mathbf{K}]\{\mathbf{u}\}=\{\mathbf{f}\}$, where $[\mathbf{K}]$ is the stiffness matrix, and $\{\mathbf{f}\}$ the vector of nodal forces. In the absence of body forces, as assumed further on, interior nodes are free from any external load, $\{\mathbf{f}\}=\{\mathbf{0}\}$. When any displacement vector $\{\mathbf{u}\}$ is prescribed, unbalanced forces $\left\{\mathbf{f}_{r}\right\}$ arise $\left\{\mathbf{f}_{r}\right\}=[\mathbf{K}]\{\mathbf{u}\}$. The equilibrium gap regularization consists in giving a penalization proportional to the sum over internal nodes of the quadratic norm of unbalanced forces

$$
\Phi_{m}^{2}=\{\mathbf{u}\}^{t}[\overline{\mathbf{K}}]^{t}[\overline{\mathbf{K}}]\{\mathbf{u}\}
$$

where ${ }^{t}$ is the transposition operator, $[\overline{\mathbf{K}}]$ the rectangular stiffness matrix left-restricted to inner nodes, and $\Phi_{m}^{2}$ corresponds to the sum of the squared norm of all equilibrium gaps of interior (and free boundary) nodes only. Additionally, a regularization for boundary nodes is introduced, namely, a third objective functional is considered

$$
\Phi_{b}^{2}=\{\mathbf{u}\}^{t}[\mathbf{L}]^{t}[\mathbf{L}]\{\mathbf{u}\}
$$

where $[\mathbf{L}]$ is an operator acting on the stress-loaded ROI boundaries [31].

The minimization of correlation residuals $\left(\Phi_{c}^{2}\right)$, equilibrium gap $\left(\Phi_{m}^{2}\right)$, and boundary fluctuations $\left(\Phi_{b}^{2}\right)$, requires the introduction of a total functional $\Phi_{t}$

$$
\left(1+w_{m}+w_{b}\right) \Phi_{t}^{2}=\widetilde{\Phi}_{c}^{2}+w_{m} \widetilde{\Phi}_{m}^{2}+w_{b} \widetilde{\Phi}_{b}^{2}
$$


where $w_{m}$ and $w_{b}$ are weights that define length scales associated with mechanical $\widetilde{\Phi}_{m}^{2}$ and boundary $\widetilde{\Phi}_{b}^{2}$ normalized residuals

$$
w_{m}=\left(\frac{|\mathbf{k}| \ell_{m}}{2 \pi}\right)^{4} \quad ; \quad w_{b}=\left(\frac{|\mathbf{k}| \ell_{b}}{2 \pi}\right)^{4}
$$

where $\ell_{m}$ and $\ell_{b}$ denote respectively bulk $(m)$ and boundary $(b)$ regularization lengths, and $\mathbf{k}$ the wavevector of the normalizing field [31]. In the following studies, 3-noded triangular elements will be considered. They are the simplest in terms of displacement interpolations, even though they are seldom used in the framework of global DIC. Let us however emphasize that the element size, when smaller than the regularization length, no longer matters.

\subsubsection{Displacement/strain measurement}

Figure 6(a) shows the reference image captured at the macroscale when the sample was already mounted in the testing machine with $F_{1}=F_{2}=0$. For macroscale observations the unstructured T3 mesh consists of 7,314 nodes with an element size of 10 pixels. Before performing a DIC measurement a resolution analysis [31] is carried out with different lengths $\ell_{m}$ (i.e., regularization length applied to the inner nodes) and $\ell_{b}$ (i.e., regularization length applied to the boundary/edge nodes) to estimate the measurement resolution. The ratio $\ell_{b} / \ell_{m}=1 / 2$ was kept constant during the whole analysis. 


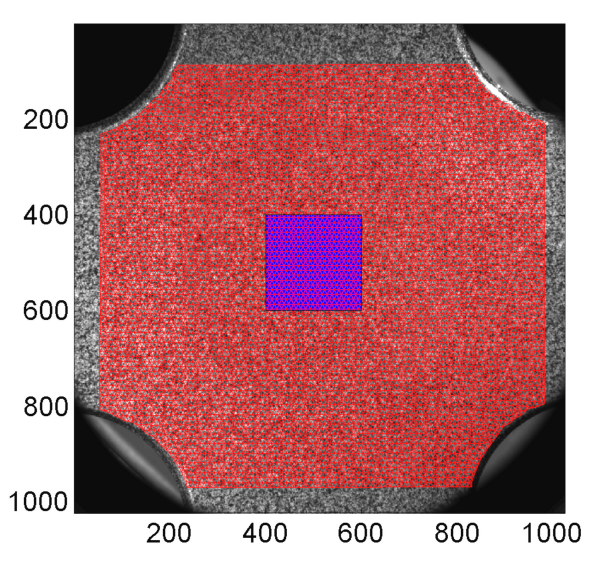

(a) 1 pixel $\leftrightarrow 48 \mu \mathrm{m}$

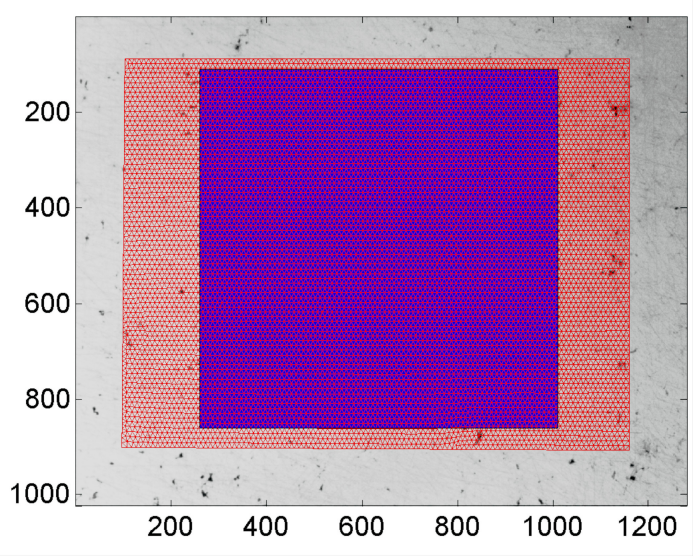

(b) 1 pixel $\leftrightarrow 6.7 \mu \mathrm{m}$

Figure 6: DIC gauges at the two scales. (a) Macroscale and (b) mesoscale DIC gauges depicted as square (blue) zones over the unstructured T3 mesh with 10 pixel elements (red). All dimensions are expressed in pixels

The standard displacement resolution in horizontal $\left(\gamma_{u}^{y}\right)$ and vertical $\left(\gamma_{u}^{x}\right)$ directions was computed. From the resolution analysis approximately equal values are reported when different regularization lengths (i.e., $32 \leq \ell_{m} \leq$ 1024 pixels) are used. Furthermore, even for modest regularization lengths (e.g., $\ell_{m}=64$ or 32 pixels), $\gamma_{u}^{x}=0.015$ pixel and $\gamma_{u}^{y}=0.012$ pixel, which is found to be adequate. This is made possible thanks to the texture quality that is better than that at the mesoscale.

Similarly, the uncertainty of the DIC analysis of the (poor) natural texture of SG cast iron is investigated (Figure 6(b)), see Appendices A and B. Even though the texture is poor 10-pixel elements are chosen (i.e., 8,749 nodes) since regularization is implemented (and $\ell_{m}$ rather than the element size will 
control the displacement uncertainty). The same resolution analysis as for macroscale pictures with two images acquired in the unloaded state. For $\ell_{m}=128$ pixels the standard displacement uncertainty is less than 0.012 pixel. When comparing the chosen regularization lengths for macro $\left(\ell_{m}=\right.$

32 pixels $)$ and meso $\left(\ell_{m}=128\right.$ pixels $)$ scale analyses, they lead to the same physical size. Conversely, the (metric) measurement resolution is four times finer for the mesoscale measurements.

Even though the geometry of the specimen is complex, the mean strain is evaluated in the central zone. Moreover one of the goals of the present study is to detect crack initiation. Hence, DIC gauges (of same physical size) are applied on macro (Figure 6(a)) and meso (Figure 6(b)) scales. From the acquired peak images corresponding to maximum and minimum load level the change of the mean strain amplitude is obtained for the chosen areas. The mean (nominal) strains are obtained by linearly interpolating the displacement fields within the DIC gauge section via least squares minimization. The mean in-plane deformation gradient components are extracted and the nominal strains are subsequently evaluated.

\section{Crack network formation for equibiaxial loading history}

\subsection{Analysis of experiment EBE-1}

\subsubsection{First loading cycle}

The material response (i.e., strain fields) of the load controlled equibiaxial experiment is first discussed. The strain fields measured at the macroscale 
are shown in Figure 7. Two characteristic loading points (see Figure 5(a)) from the first cycle are chosen. The strain fields shown in Figures 7(a) and 7(b) correspond to the maximum load level (i.e., $40 \mathrm{kN}$ ).

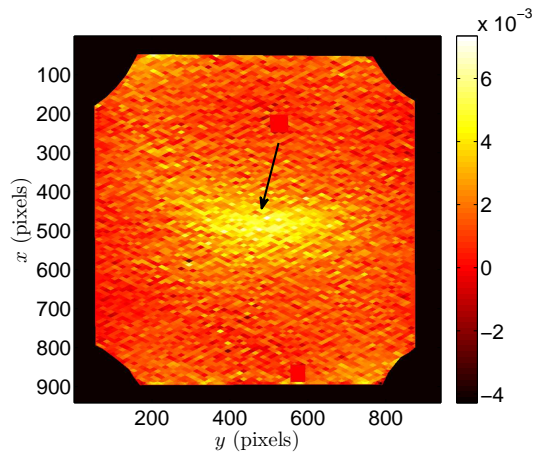

(a) Point $1, \epsilon_{x x}$

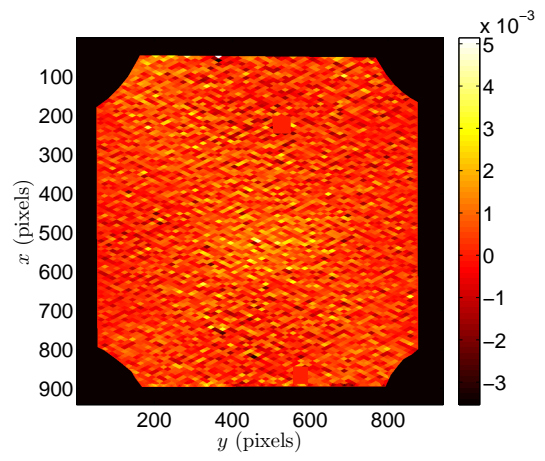

(c) Point 2, $\epsilon_{x x}$

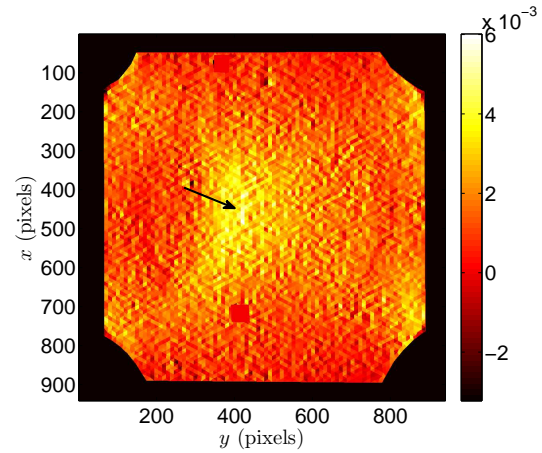

(b) Point 1, $\epsilon_{y y}$

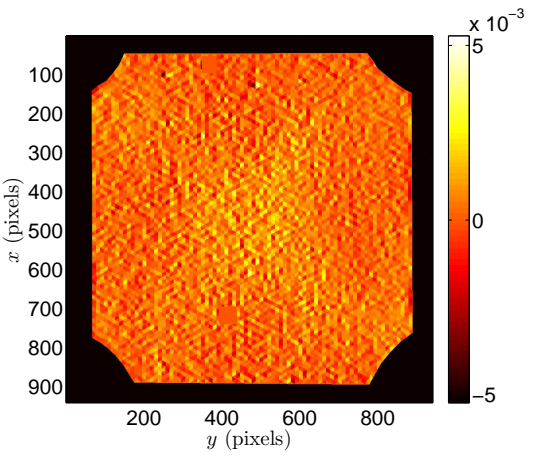

(d) Point 2, $\epsilon_{y y}$

Figure 7: Measured strain fields for first cycle at the macroscale in the $x$ (left) and $y$ (right) directions of experiment EBE-1. (a) and (b) correspond to loading point 1 (see Figure 5(a)), (c) and (d) correspond to loading point 2. The arrows indicate the strained bands. 1 pixel $\leftrightarrow 48 \mu \mathrm{m}$

The highest strain levels occur in the thinned central part of the cruciform sample. Furthermore, the specimen design satisfies the condition that fillets 
connecting the loading arms experience lower strain levels when compared to the gauge zone. In the center of the specimen two major strained bands are observed in both loading directions (see arrows in the figures).

Figures $7(\mathrm{c})$ and $7(\mathrm{~d})$ show the measured strain fields for the unloaded point of the first cycle. The strain fluctuations captured for the unloaded stage of the first cycle are one order of magnitude higher than the standard strain resolution (see Appendix B). Consequently, the strain fluctuations are believed to be physical.

For the same loading steps mesoscale strain fields are presented in Figure 8 . For the first loading point (Figure $8(\mathrm{a})$ and $8(\mathrm{~b})$ ) the strained bands are observed in the thinned gauge zone. Furthermore, the bands of the $\epsilon_{x x}$ field (see Figure $8(\mathrm{a})$ ) are perpendicular to the $x$-axis. Conversely, strained bands parallel to the $x$-axis are reported from $\epsilon_{y y}$ maps. At the final stage of the first cycle (see Figure 8(c) and 8(d)) contours of the previously reported strain bands are perceptible. The measured fluctuations after the first cycle are ten times higher than the strain resolution evaluated for the mesoscale pictures. It implies that after the first cycle permanent strains are accumulated. 


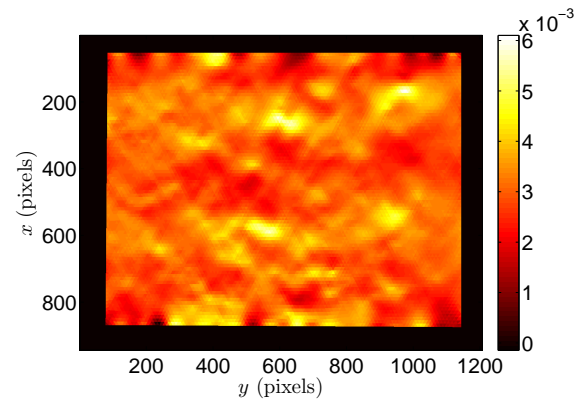

(a) Point $1, \epsilon_{x x}$

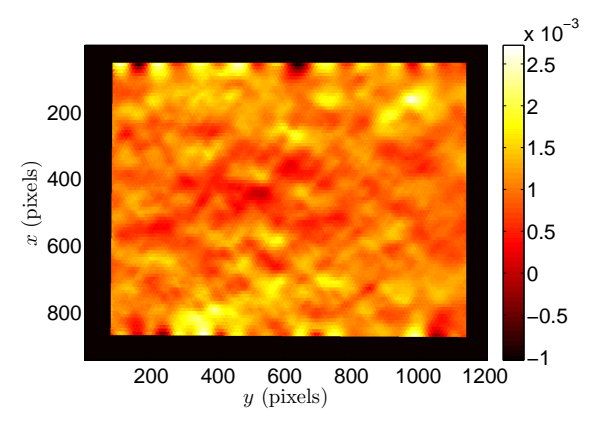

(c) Point 2, $\epsilon_{x x}$

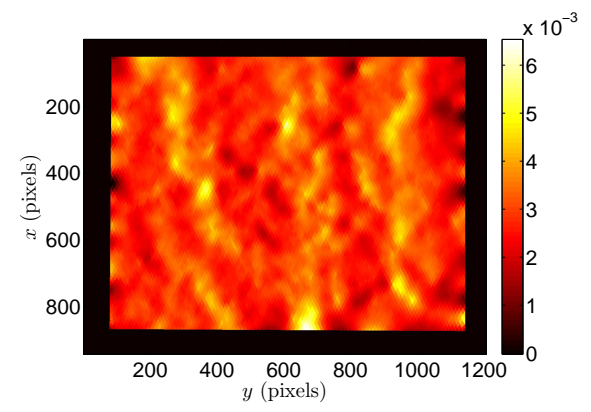

(b) Point 1, $\epsilon_{y y}$

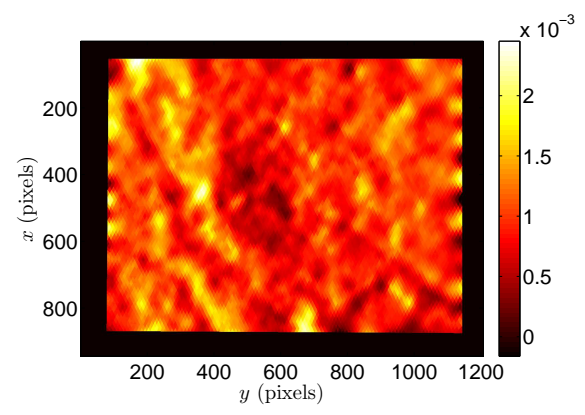

(d) Point 2, $\epsilon_{y y}$

Figure 8: Measured strain fields for the first cycle at the mesoscale in the $x$ (left) and $y$ (right) directions of experiment EBE-1. (a) and (b) correspond to loading point 1 (see Figure 5(a)), (c) and (d) correspond to loading point 2. 1 pixel $\leftrightarrow 6.7 \mu \mathrm{m}$

Figure 9(a) shows the mean strain history at the mesoscale for the first cycle. It is noted that mean eigen strains (Figure 9(b)) virtually coincide with the strain components in the loading direction. However, the strain levels are not equal. Nonlinear strains are detected after $0.15 \%$. In the first regime, the strain levels for the two eigen components follow the same path even though microcracks start to appear. The difference reported between the mean strain levels in the loading direction and the eigen strains is due 
to the heterogeneous nature of the microcrack distribution. Plasticity effects are also observed since the strain level corresponding to full unloading does not vanish.

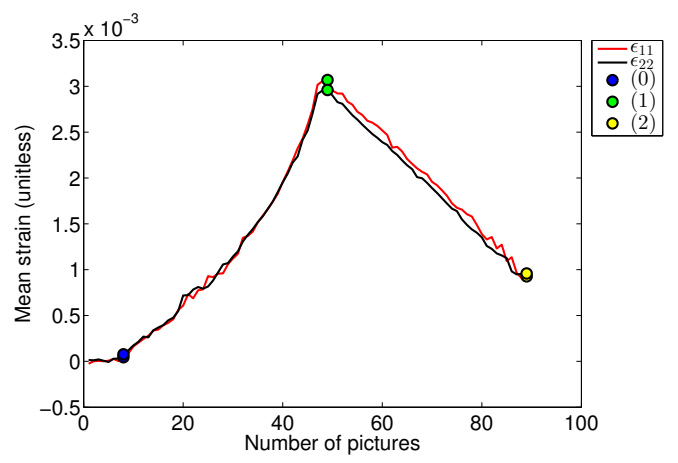

(a)

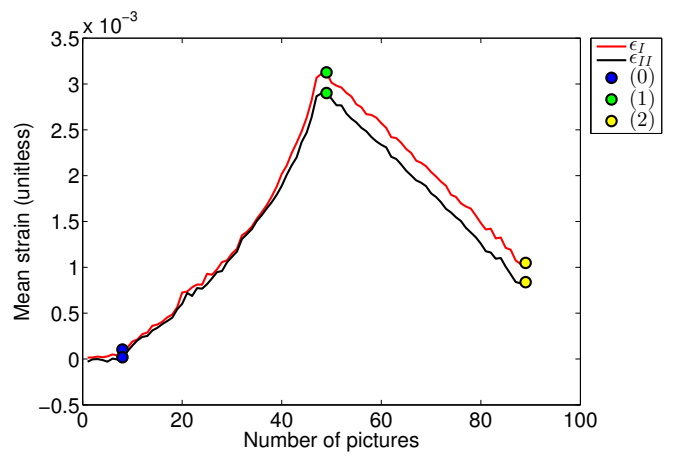

(b)

Figure 9: Strain histories of the first cycle of EBE-1 experiment $\left(F_{\max }=40 \mathrm{kN}\right)$. (a) Mean strains $\epsilon_{11}$ and $\epsilon_{22}$ in the loading directions of the sample. (b) Mean eigen strains. Blue, green and yellow circles correspond to the three characteristic points of equibiaxial experiments (see Figure 5(a))

Figure 10 illustrates the correlation residual $\varphi_{c}(\mathbf{x})$ maps obtained from RT3-DIC between the reference configuration and that corresponding to the 
maximum load level of the first cycle at the mesoscale. A high value of the residuals indicates a change in the surface texture that cannot be accommodated by a displacement field with the restrictions attached to those considered in the DIC analysis (e.g., discretization over the chosen mesh, regularity). In the correlation residual map (Figure 10(a)) short microcracks are clearly visible (see arrows in the figure). They are randomly distributed on the surface of the sample, but are not yet forming a crack network. However, in the right bottom and top parts longer cracks are observed. Last, the concentric rings correspond to machining defects.

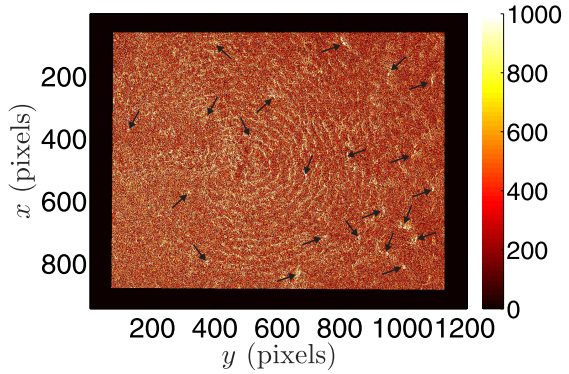

(a)

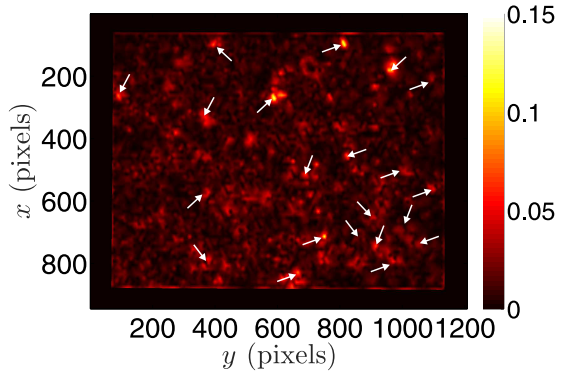

(b)

Figure 10: Mesoscale (a) correlation (in gray level) and (b) mechanical residual maps corresponding to maximum load level (i.e., $F_{1}=F_{2}=40 \mathrm{kN}$ ) for the first cycle of EBE-1 experiment. The arrows indicate the detected cracks. 1 pixel $\leftrightarrow 6.7 \mu \mathrm{m}$

The mechanical residuals (Figure 10(b)) also show similar trends (see arrows in the figure). Most of the crack branches are found in the right part of the map, with the most opened ones in the right bottom corner. It is necessary to emphasize that correlation residuals indicate more clearly the 
crack paths since they are obtained pixel-wise while the mechanical residuals are calculated at nodal (i.e., element) levels.

\subsubsection{Strain history}

With the initiation and propagation of cracks the strain levels are expected to increase and one of the criteria to identify microcrack growth consists in extracting the strain amplitudes (i.e., difference of maximum and minimum strains over one cycle). The mean strains are calculated with the DIC gauge in order to determine the strain path. Figure 11 shows the material response with the number of cycles. The graph shows smooth episodes interrupted by sudden jumps (discontinuities). Eight of them are seen. From the two mean eigen components the jumps are more apparent on the $\epsilon_{I}$ levels. 


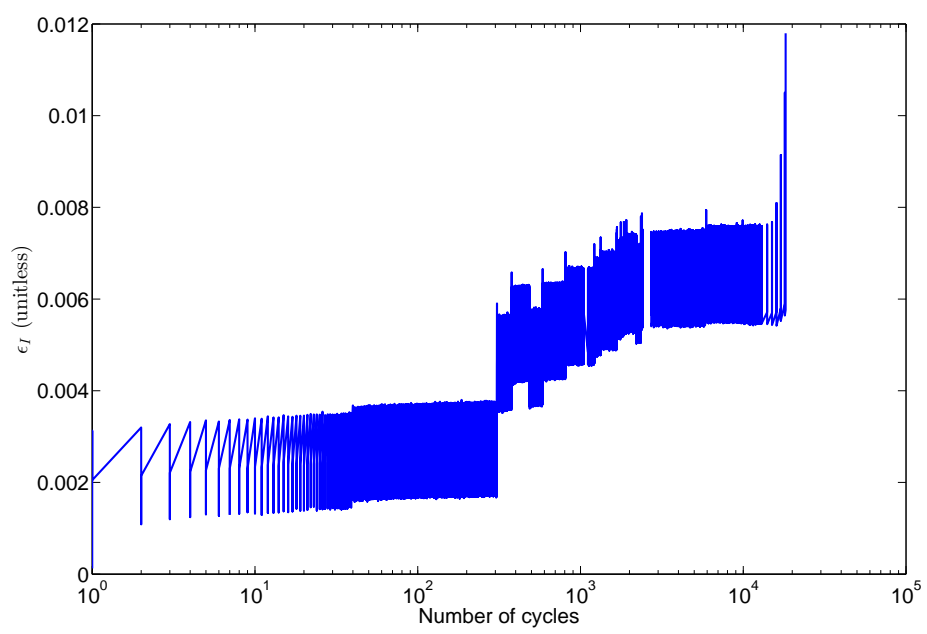

(a)

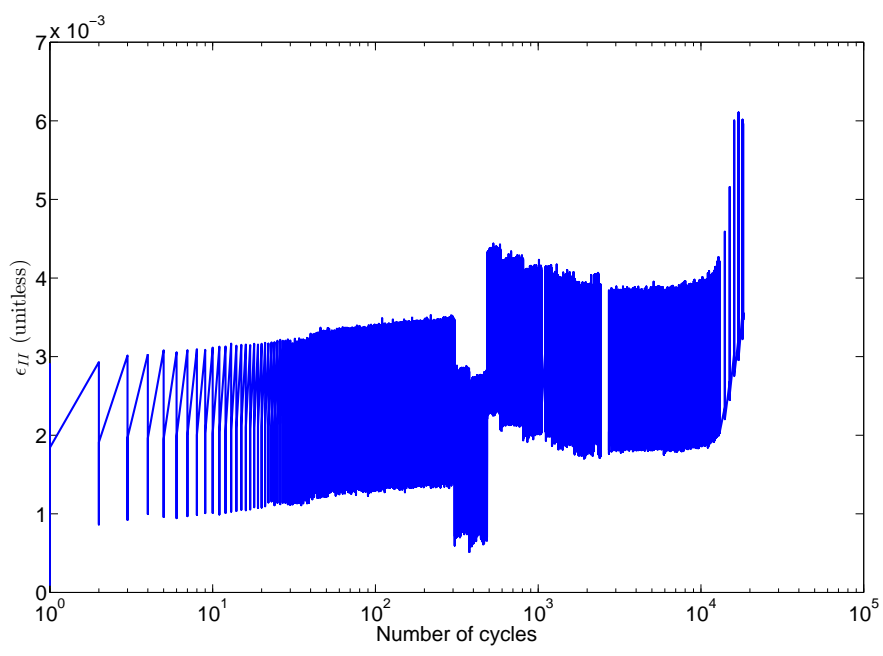

(b)

Figure 11: Measured (a) $\epsilon_{I}$ and (b) $\epsilon_{I I}$ histories with respect to the number of cycles of experiment EBE-1.

The first increase in $\epsilon_{I}$ and simultaneous decrease of $\epsilon_{I I}$ occurs because the experiment was interrupted, which caused a change in the mean strain 
level. However, it did not result in a change of the strain amplitudes (see Figure 12) of the mesoscale observation. From the residual maps, a growth of the crack network is observed, as discussed below.

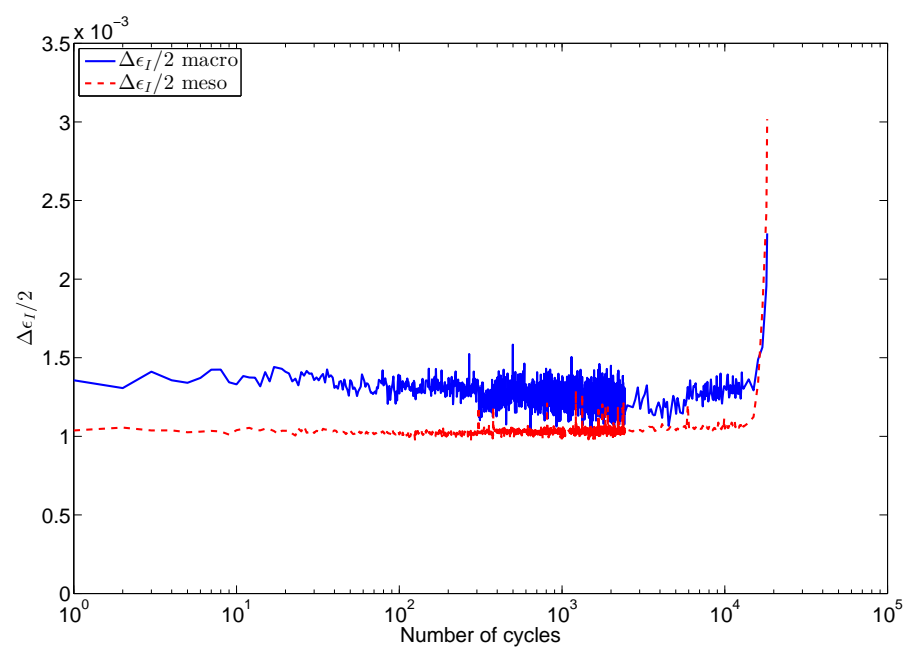

(a)

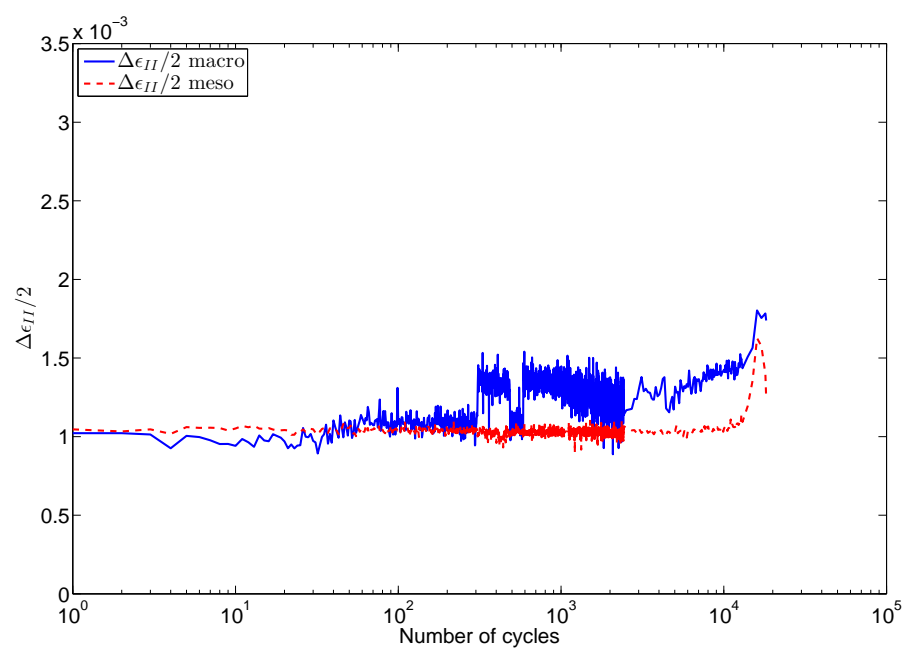

(b)

Figure 12: Measured amplitudes for (a) $\epsilon_{I}$ and (b) $\epsilon_{I I}$ at macro- and mesoscales of experiment EBE-1 
Figure 12 shows the strain amplitude history observed on the two sides of the sample. When comparing the mean strain amplitudes with the number of cycles the macroscale observations are more fluctuating than the mesoscale data. However, this fact is not surprising since less information is available at the macroscale (i.e., the physical size of the DIC gauge is identical at macro and mesoscales, see Figure 6). The $\Delta \epsilon_{I I}$ amplitudes (Figure 12(b)) are very close for the two scales. This is not the case for $\Delta \epsilon_{I}$ (Figure 12(a)) where the gap between the two scales is higher and progressively decreases. The difference (initially of about $0.03 \%$ ) may be an indication of spurious flexure (Appendix C). For the first 2,500 loading cycles strain amplitudes are calculated for each cycle. Thereafter, every 100th cycle is taken into account. This reduced sampling rate gives the fake impression of reduced fluctuations, and, in a similar fashion, the $\log$ scale used for the number of cycles may suggest increasing fluctuations (see Figure 12) whereas this is not justified by a more systematic analysis.

After 7,500 loading cycles the amplitudes $\Delta \epsilon_{I}$ and $\Delta \epsilon_{I I}$ at the mesoscale gradually increase until cycle 15,000 is reached. At this point an increasing gap of $\Delta \epsilon_{I}$ and $\Delta \epsilon_{I I}$ is noted. Macroscale observations follow the same trend for both eigen strains. It is necessary to emphasize differences between macroand mesoscale strain amplitudes after the increase is reported. $\Delta \epsilon_{I}$ at the mesoscale reaches higher values, which may be caused by spurious flexure of the sample. 


\subsubsection{Crack network}

In order to detect the crack network growth, normalized mechanical (i.e., $\tilde{\Phi}_{m}$ ) and correlation (i.e., $\hat{\Phi}_{c}$ ) residuals are considered. Cracks initiate at the very first loading cycle. Mesoscale observations better detect the initiation and early propagation stages in the thinned gauge zone of the specimen. Hence, these residuals will be used (see Figure 13(a)). Both residuals exhibit less fluctuations than the mean strains (see Figure 12). The residuals follow the same trend with an increase after the first loading cycle. This leads to the conclusion that cracks start to grow in each loading cycle (i.e., material is more affected by damage). When comparing the two residuals for this

case it is observed that $\hat{\Phi}_{c}$ is slightly more sensitive to detect changes on the surface of the material. 


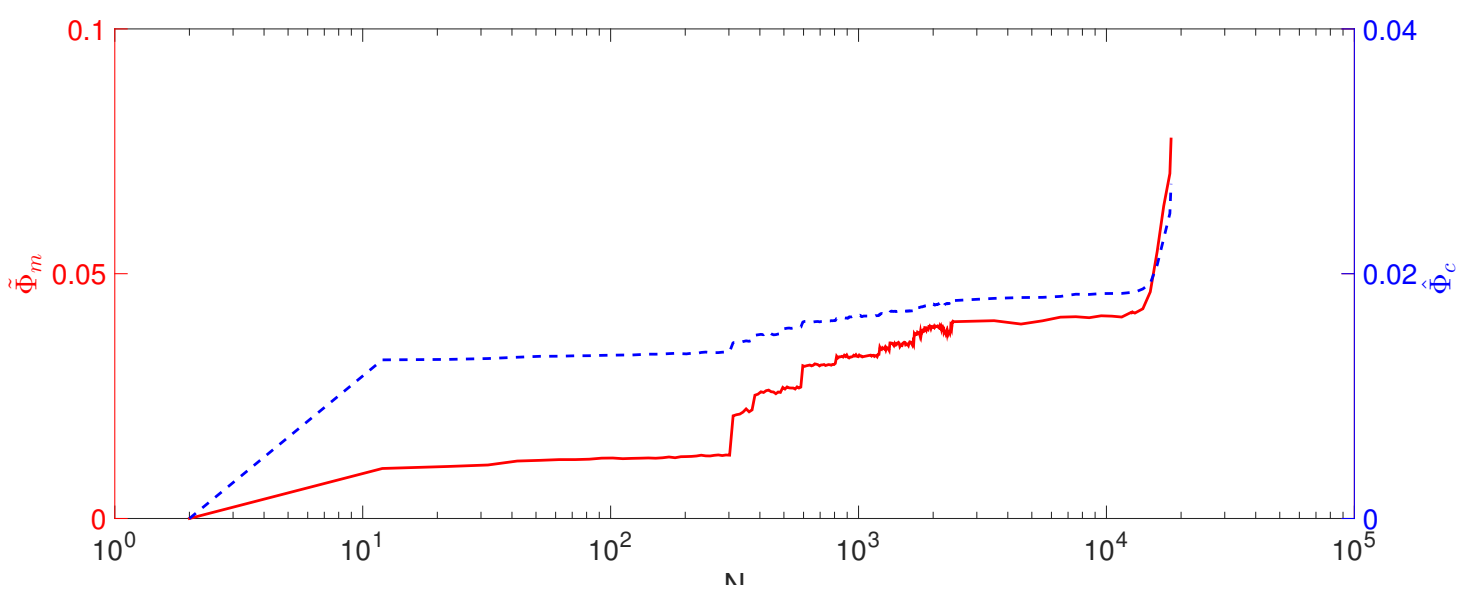

(a)

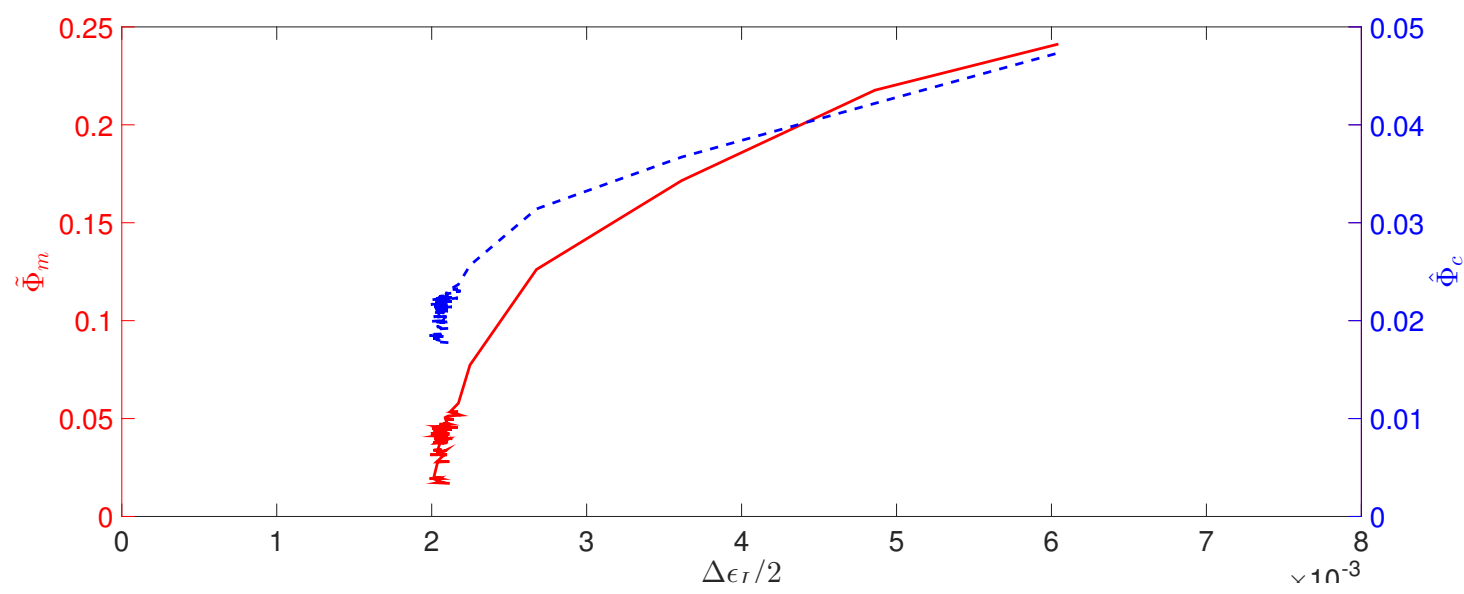

(b)

Figure 13: (a) Change of mesoscale mechanical $\tilde{\Phi}_{m}$ and normalized correlation residuals $\hat{\Phi}_{c}$ with the number of cycles of experiment EBE-1. (b) Corresponding change of $\tilde{\Phi}_{m}$ and $\hat{\Phi}_{c}$ with respect to the strain amplitude $\Delta \epsilon_{1} / 2$

Figure 13(b) illustrates the changes of both residuals as functions of the maximum eigen strain amplitude measured in the gauge section. The variations of the residuals are more sensitive until the 125-th cycle (see Figure 12) 
than the corresponding strain amplitude $\Delta \epsilon_{I I}$. In this part of the fatigue experiment strain amplitudes have fluctuations that could be attributed to noise. In the second part (i.e., beyond 125 cycles) the strain variations start to change significantly, which is caused by faster growing cracks.

Figure 14 shows the maps of correlation and mechanical residuals for different numbers of cycles. The cracks captured for cycle 1,600 (Figure 14(a) and (b)) are longer than at the first cycle (Figure 10). Moreover, they correspond to a significant increase of the level of global residuals as shown in Figure 13. Residuals for cycle 1,600 confirm that fact since from cycle 150 to 1,600 more cracks initiate than from cycle 1,600 to 12,000 (i.e., see the correlation residuals shown in Figure 14(a)). Furthermore, between cycle 1,600 (Figure 14(a) and (b)) and 12,000 (Figure 14(c) and (d)) the crack network growth is stabilized. Accordingly, the correlation and mechanical residuals do not increase significantly (Figure 13). 224 cycles before failure of the cruciform specimen a dominant macrocrack is detected from the residual fields (Figure 14(e) and (f)). Consistently, the same phenomenon is observed for the residual histories (Figure 13). The residual levels reported for cycle 18,000 are approximately twice as large as those of cycle 12,000. 


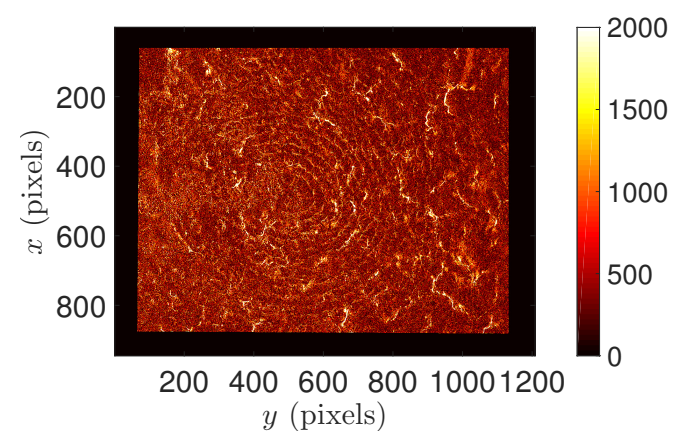

(a) Cycle 1,600

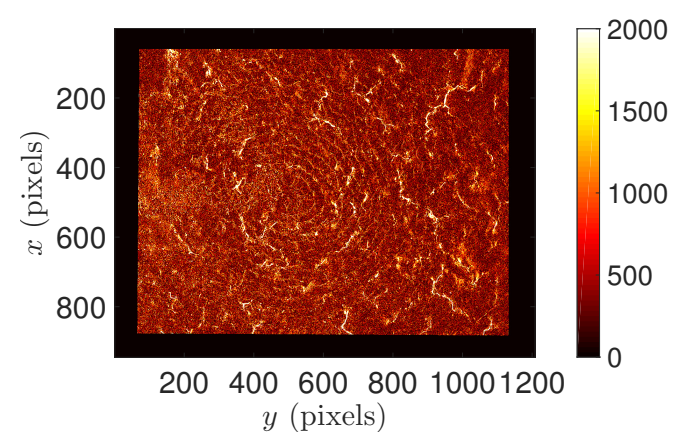

(c) Cycle 10,000

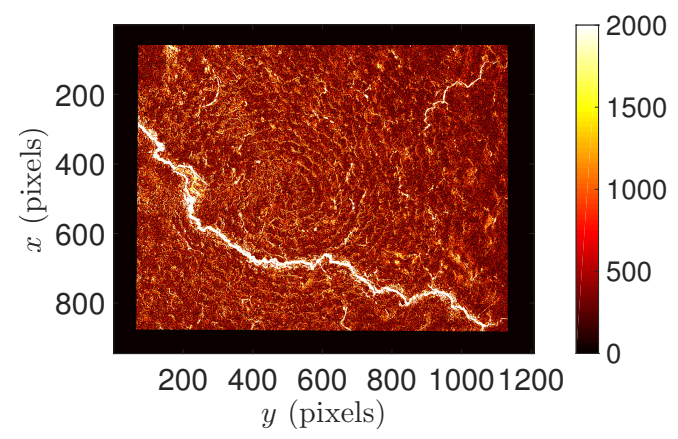

(e) Cycle 18,000

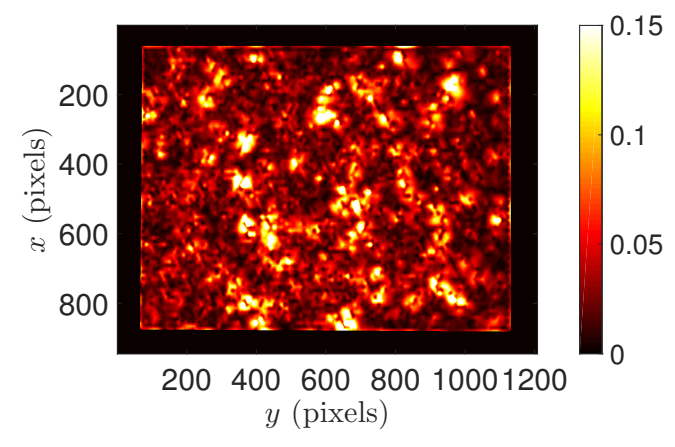

(b) Cycle 1,600

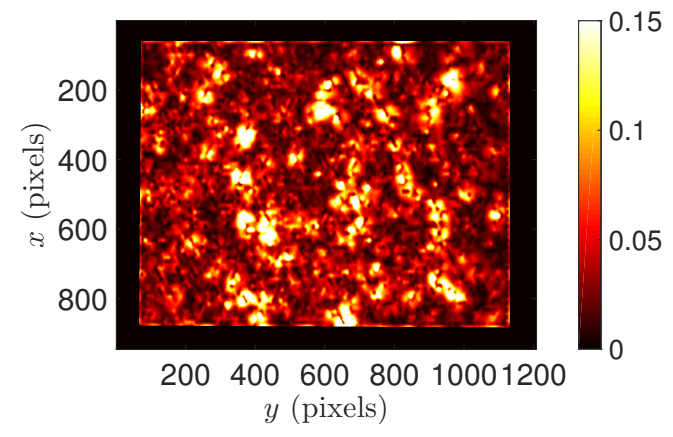

(d) Cycle 10,000

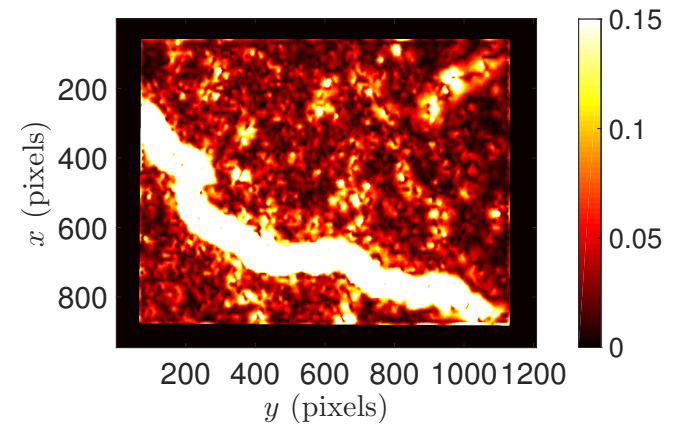

(f) Cycle 18,000

Figure 14: Mesoscale (gray level) correlation (left) and mechanical (right) residual maps corresponding to the maximum applied load level for different number of cycles of experiment EBE-1. 1 pixel $\leftrightarrow 6.7 \mu \mathrm{m}$ 
For this test, it is shown that the correlation and mechanical residuals are much more sensitive to the development of damage and crack growth regime than the mean strains. This is partly due to the fact that crack initiation takes place at the first loading cycle. It will be shown (for the following test case) that for a less severe loading, the mean strains play a more significant role. When observing the correlation residuals in Figures 10 and 14 concentric marks are observed. They have been induced upon machining the caps. Hence, they have no mechanical background. Even though the residuals arise over these machining marks their appearance is not influencing the quality of the results (i.e., measured strain maps). While evaluating the strain fields (see Figure 15) for the maximum loading no artifacts caused by the machining marks are reported. 


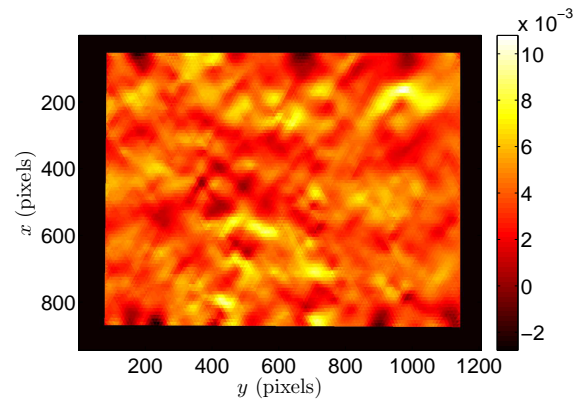

(a) Cycle 1,000, $\epsilon_{x x}$

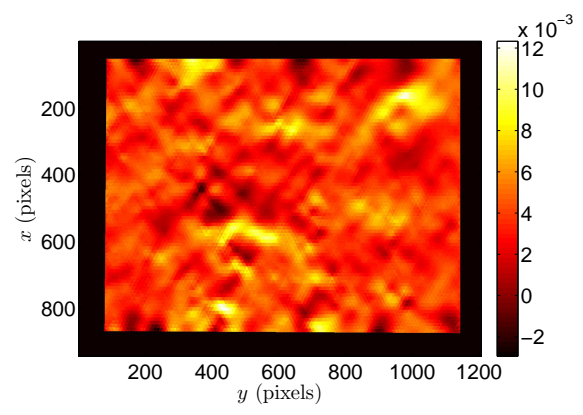

(c) Cycle $10,000, \epsilon_{x x}$

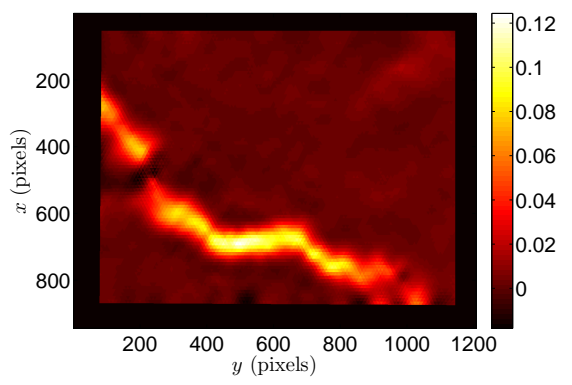

(e) Cycle 18,000, $\epsilon_{x x}$

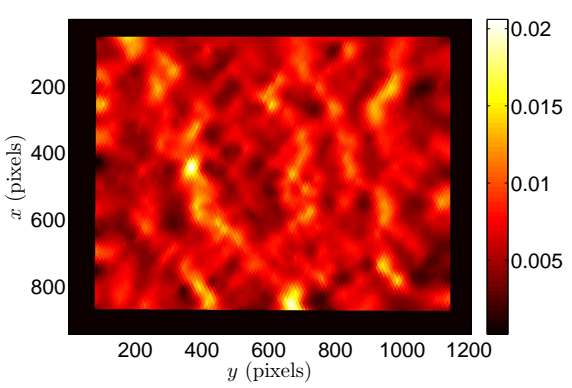

(b) Cycle 1,000, $\epsilon_{y y}$

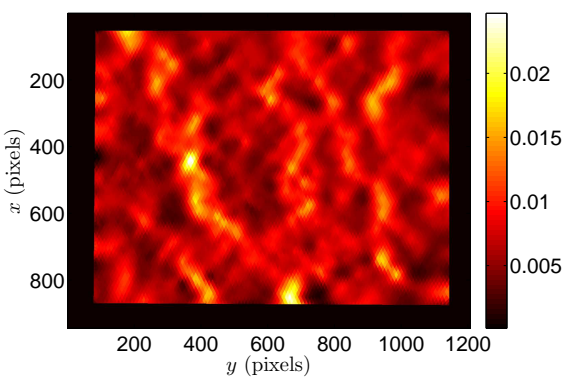

(d) Cycle 10,000, $\epsilon_{y y}$

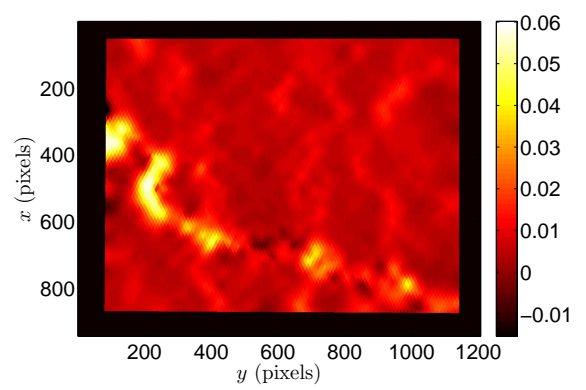

(f) Cycle $18,000, \epsilon_{y y}$

Figure 15: Measured strain fields corresponding to the maximum applied load level at the mesoscale in the $x$ (left) and $y$ (right) directions for different numbers of cycles of experiment EBE-1. 1 pixel $\leftrightarrow 6.7 \mu \mathrm{m}$ 


\subsection{Microcrack initiation for EBE-2 test}

The second equibiaxial fatigue test was cycled in tension with a maximum load equal to $20 \mathrm{kN}$ (i.e., half that of EBE-1 test) for $10^{6}$ cycles. After reaching this stage, the experiment was stopped and the maximum load level was increased to $F_{1}^{\max }=F_{2}^{\max }=25 \mathrm{kN}$. The sample broke for a total number of $1.5 \times 10^{6}$ cycles.

Figure 16 shows the mean eigen strain histories obtained for the DIC gauge at the mesoscale for the first cycle. The maximum strain level equals $0.1 \%$.

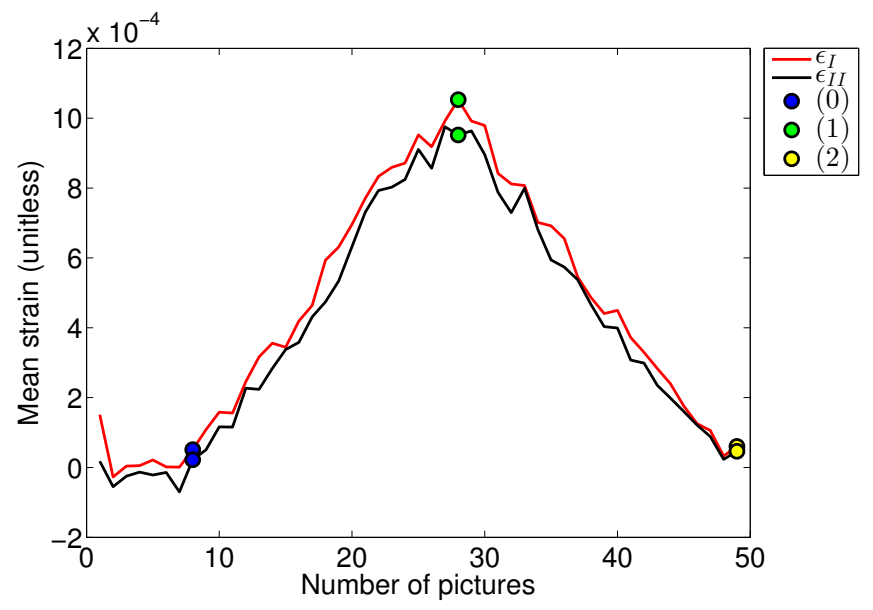

Figure 16: Mean eigen strains for the first cycle of equibiaxial experiment EBE-2 (i.e., $F_{\max }=20 \mathrm{kN}$ ) at the mesoscale. Blue, green and yellow circles correspond to the three characteristic points of Figure 5(a)

When compared with the strain level of the previous test (Figure 9) a linear response is observed. A very good coincidence is noted between the two measured eigen strains. As opposed to EBE-1 experiment (where $F^{\text {max }}=40$ 
$\mathrm{kN}$ ) the unloading in the first cycle leads to the same level as at the reference point. From this result it can be concluded that the central part of the sample is not damaged, which makes it more challenging to detect microcracks.

The correlation and mechanical residual maps are shown in Figure 17, for the first loading cycle. Uniform correlation residuals (Figure 17(a)) show a very good match between the reference and corrected deformed image, and mechanical residuals (Figure 17(b)) also display rather low fluctuations. These observations confirm the absence of damage during the first loading cycle.

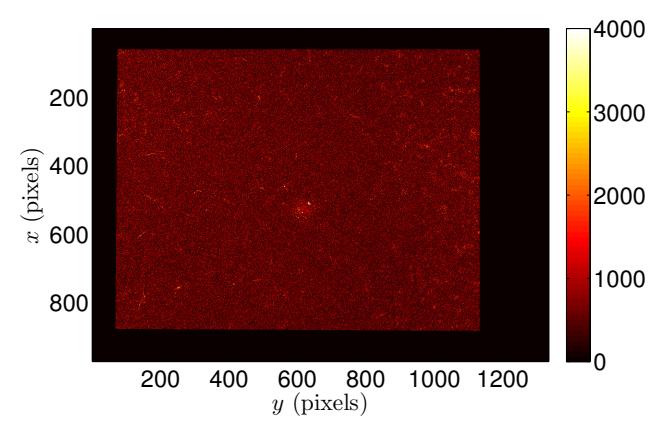

(a)

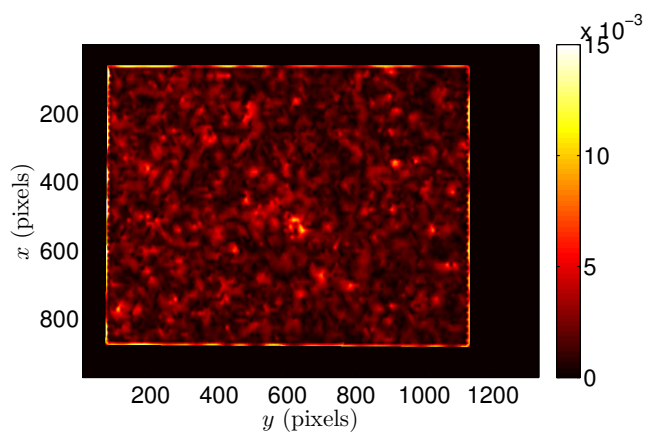

(b)

Figure 17: Mesoscale (a) correlation (in gray levels) and (b) mechanical residual maps corresponding to a maximum applied load $\left(F_{1}=F_{2}=20 \mathrm{kN}\right)$ for the first cycle of experiment EBE-2. 1 pixel $\leftrightarrow 6.7 \mu \mathrm{m}$

Following the fatigue experiment along the cycles, Figure 18 shows that $\tilde{\Phi}_{m}$ and $\hat{\Phi}_{c}$ global residuals are proportional to each other, over the whole range of $1.5 \times 10^{6}$ cycles. Both quantities can therefore be used for detection purposes. 


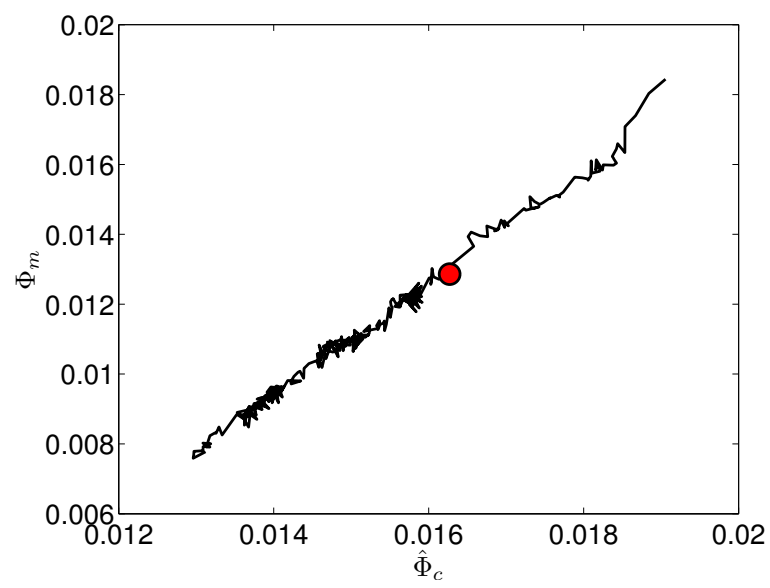

Figure 18: Correlation between mechanical $\left(\tilde{\Phi}_{m}\right)$ and normalized correlation $\left(\hat{\Phi}_{c}\right)$ residuals from whole series of images acquired in experiment EBE-2 at mesoscale. The red circle denotes the point where the applied load level has been increased

The mean eigen strains can also be studied (Figure 19(a)) at macro- and mesoscales. In the present case, fluctuations of $\Delta \epsilon_{I}$ are studied on both scales; they are much higher than those observed on the residuals and no systematic trend is detected in the first million cycles (Figure 19(a)). Figure 19(b) shows the change of correlation and mechanical residuals as functions of the maximum eigen strain amplitude extracted at the mesoscale. For the first $10^{6}$ cycles strain fluctuations of the order of $0.02 \%$ are observed. In contrast, correlation and mechanical global residuals exhibit a steady increase. In the first million of cycles $\left(F^{\max }=20 \mathrm{kN}\right)$, it is very difficult to infer the presence of a crack network from the strain amplitudes. In the final part of the test, where the maximum load is raised to $F^{\max }=25 \mathrm{kN}$, a significant increase of $\Delta \epsilon_{I}$ is noted. 


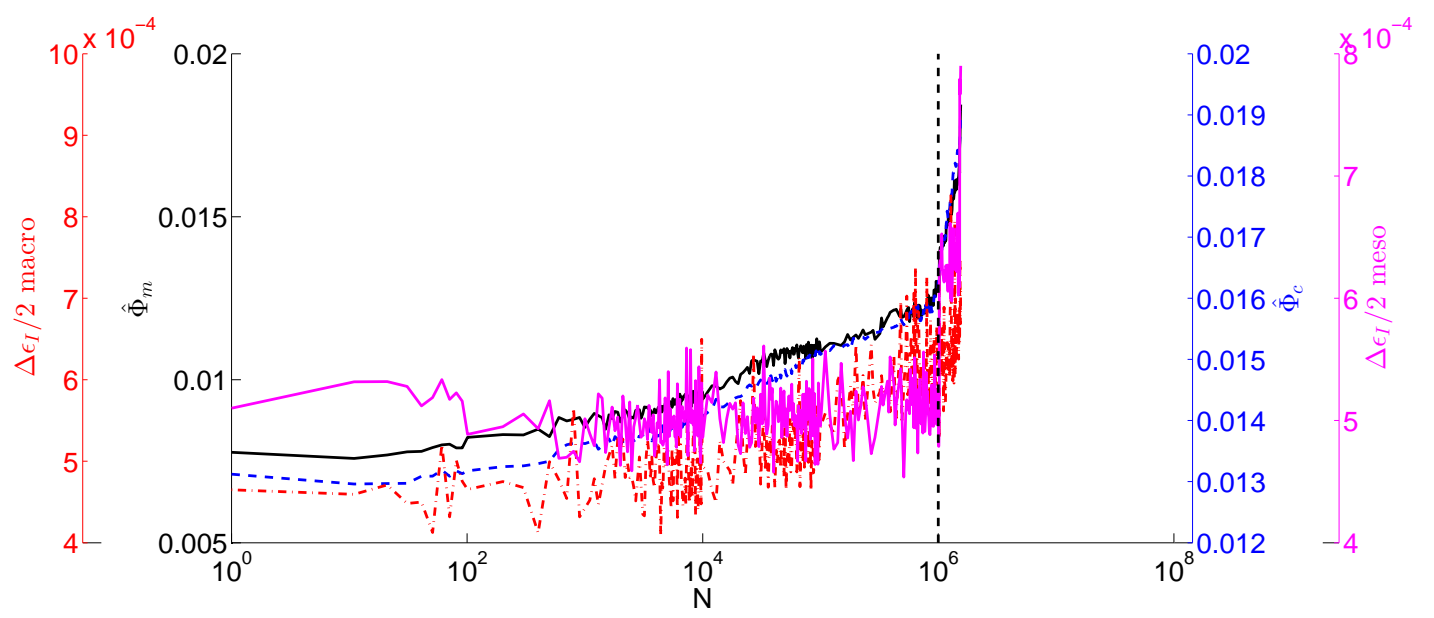

(a)

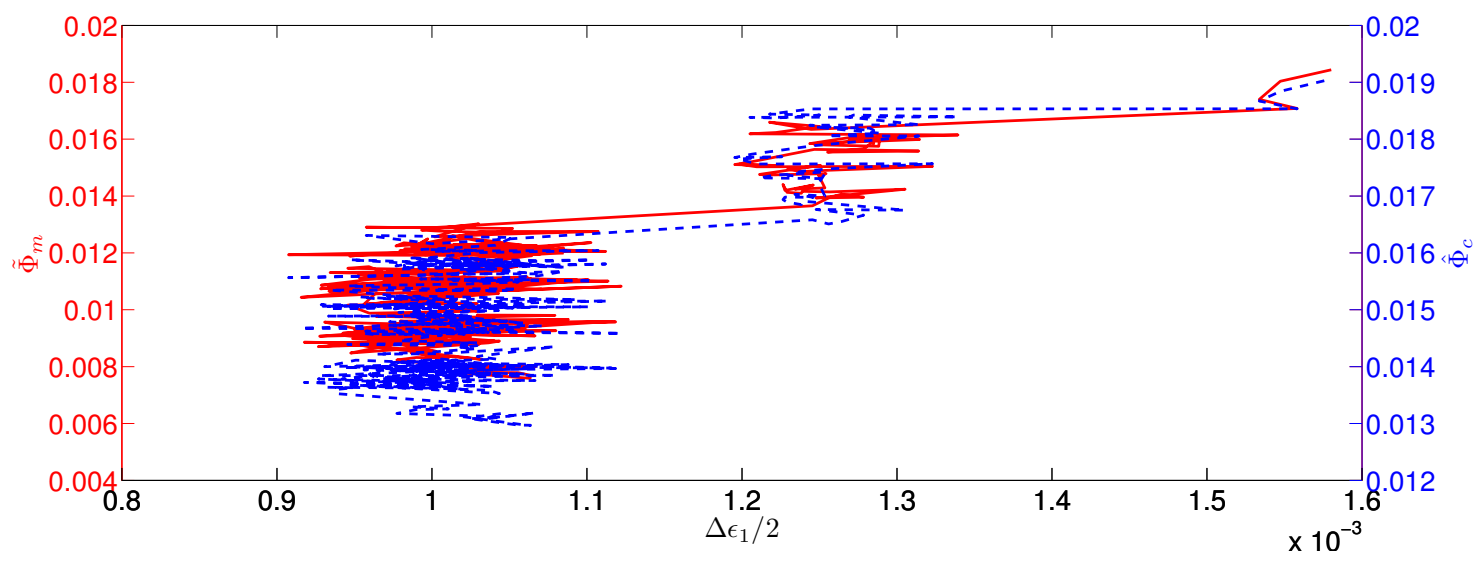

(b)

Figure 19: (a) Changes of mesoscale mechanical $\tilde{\Phi}_{m}$ and normalized correlation residuals $\hat{\Phi}_{c}$ with the number of cycles of experiment EBE-2. For comparison purposes $\Delta \epsilon_{I}$ are reported at macro- and mesoscales. The vertical dashed line shows when the applied load has been increased. (b) Changes of $\tilde{\Phi}_{m}$ and $\hat{\Phi}_{c}$ with the strain amplitude $\Delta \epsilon_{I}$ at the mesoscale

Figure 20 shows the correlation residual maps. Small discontinuities are 
detected with higher values of the residuals for cycle 10,000. As the sample is cycled the microcracks start to grow. A significant increase of their population is reported from cycles 10,000 to 50,000. Whereas in the previous test, one big crack was formed before the specimen broke, this is not the case in the present test. On the image acquired after $1.55 \times 10^{6}$ cycles a group of randomly distributed microcracks has initiated. The failure was sudden and without stable coalescence of a macrocrack. 


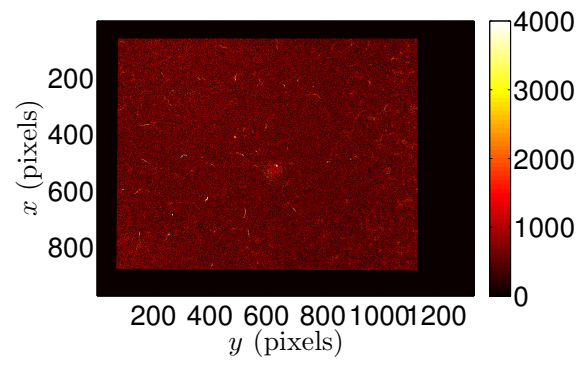

(a) Cycle 1,000

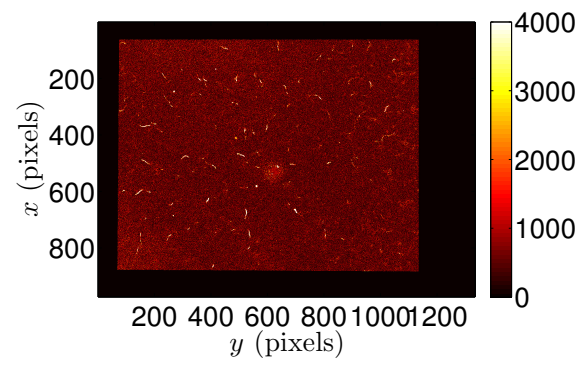

(c) Cycle 50,000

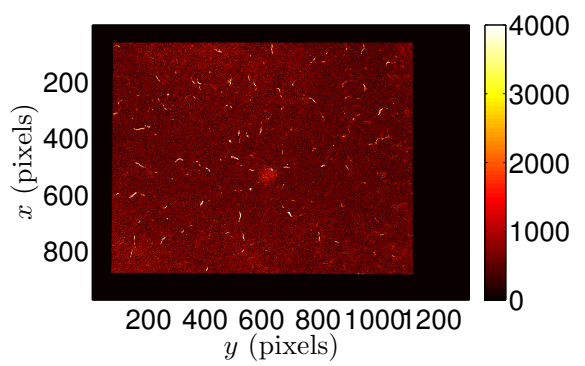

(e) Cycle 500,000

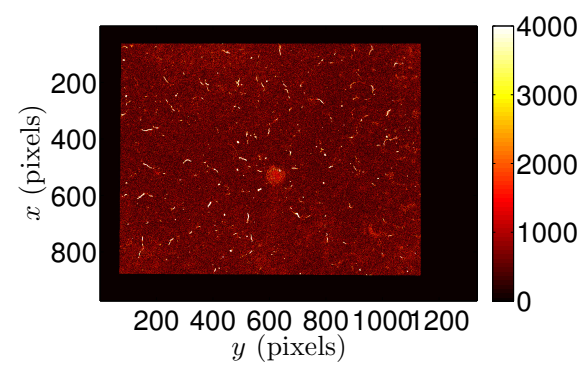

(g) Cycle 1,250,000

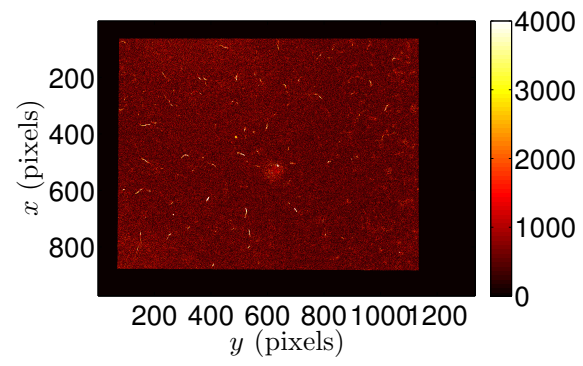

(b) Cycle 10,000

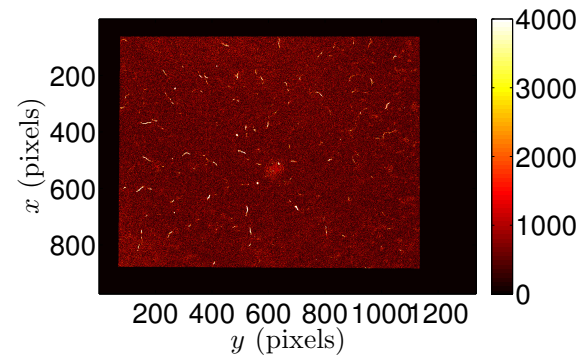

(d) Cycle 100,000

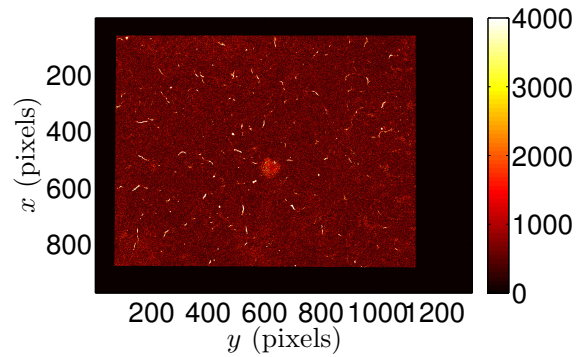

(f) Cycle 1,000,000

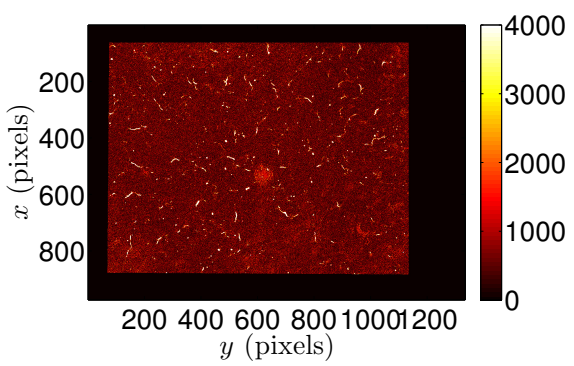

(h) Cycle 1,550,000

Figure 20: Mesoscale (gray level) correlation residual maps for different numbers of cycles of experiment EBE-2. 1 pixel $\leftrightarrow 6.7 \mu \mathrm{m} 38$ 


\section{Crack network formation for square loading}

Square loading histories lead to more complex material responses than equibiaxial loadings [32], be they of constant amplitude as considered herein, or with a progressive increase in amplitude, or "snail" loading (with reference to spiraling loading path). Let us stress that the maximum load level is identical in the two following cases, SBE-1 and 2 respectively, as in the previously studied EBE-1 and 2 test cases. However, in the square loading

path, the equivalent stress levels are higher (Table 2). The aim of the present analysis is to investigate the sensitivity of the fatigue response of SG cast iron to such a characteristics of the loading history.

\subsection{Analysis of experiment $S B E-1$}

\subsubsection{First loading cycle}

Figure 21 shows the strain fields on the macroscale. First the sample is loaded in the $x$-direction while the load in $y$-direction is equal to zero. Hence, the strains in the loading direction are positive (Figure 21(a)) and negative in the $y$-direction (Figure $21(\mathrm{~b})$ ) in the gauge zone. High $\epsilon_{x x}$ strain gradients in the $y$-direction are observed in the thinned center part of the sample. Moreover, strain bands also occur outside of the gauge zone. They are oriented toward the arm fillets. 


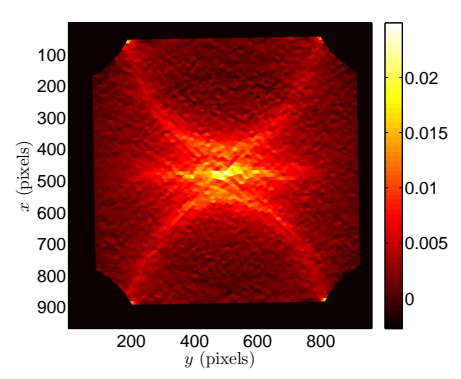

(a) Point $1, \epsilon_{x x}$

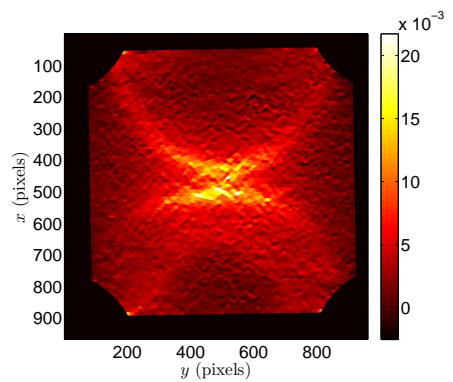

(c) Point 2, $\epsilon_{x x}$

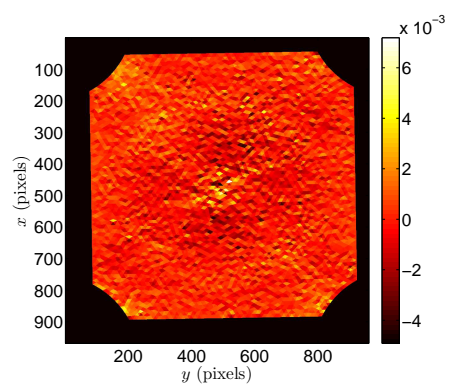

(e) Point $3, \epsilon_{x x}$

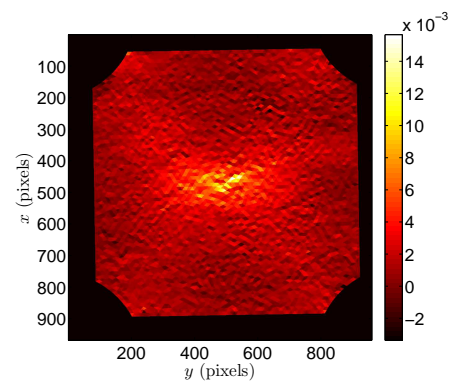

(g) Point 4, $\epsilon_{x x}$

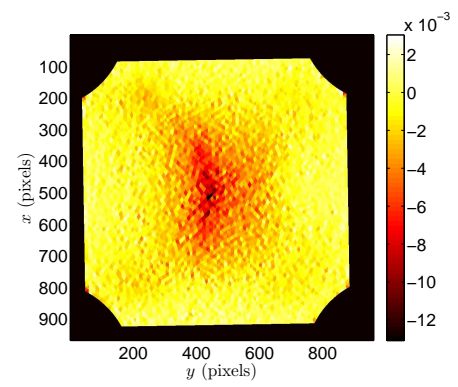

(b) Point $1, \epsilon_{y y}$

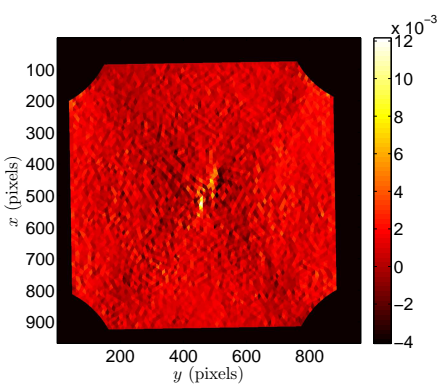

(d) Point 2, $\epsilon_{y y}$

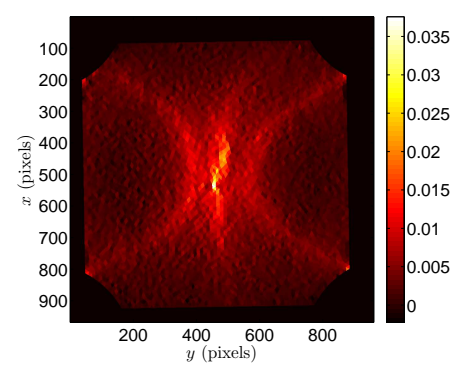

(f) Point 3, $\epsilon_{y y}$

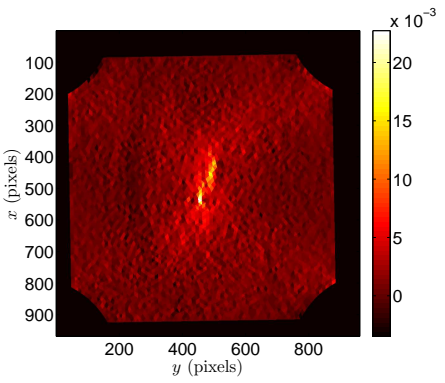

(h) Point 4, $\epsilon_{y y}$

Figure 21: Measured strain fields for first 49 cle at the macroscale in the $x$ (left) and $y$ (right) directions of experiment SBE-1. (a) and (b) correspond to loading point 1 (see Figure 5(b)), (c) and (d) to loading point 2, (e) and (f) to loading point 3, (g) and (h) to loading point $4 . \quad 1$ pixel $\leftrightarrow 48 \mu \mathrm{m}$ 
The second loading point of the square path corresponds to the first one of the equibiaxial loading path. However, the measured strain maps are different. The $\epsilon_{x x}$ strain field (Figure 21(c)) remains close to the first loading step. From Figure 21(d) it is noted that extensional strain localization occurs in the gauge area while two contraction bands at $45^{\circ}$ with respect to the loading direction are formed. Furthermore, for the same maximum applied load level (i.e., $40 \mathrm{kN}$ ) the square loading regime yields higher strain levels when compared to the equibiaxial test case (see Figure 7).

In the third loading step the $x$-axis is unloaded while the load level in the $y$-axis remains unchanged with respect to the previous step. A mostly homogeneous $\epsilon_{x x}$ field (Figure 21(e)) is observed with two extensional strain bands formed at $45^{\circ}$ with respect to the loading axis. However, the $\epsilon_{y y}$ strain field (Figure 21(f)) has a pattern that is very close to that observed in the first loading step for the $\epsilon_{x x}$ strain field (Figure 21(a)).

The strain maps corresponding to the last loading step are shown in Figures 21(g) and 21(h). Both strain components have a highly strained pattern in the gauge zone. The strain levels in the thinned center are fifty times higher than the standard strain resolution. Consequently, macrocracks have initiated.

In order to evaluate the material response at the mesoscale the measured strain maps are presented in Figure 22. 


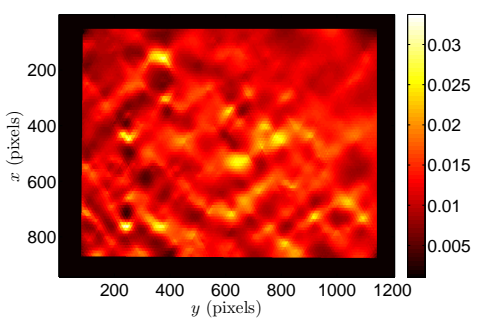

(a) Point $1, \epsilon_{x x}$

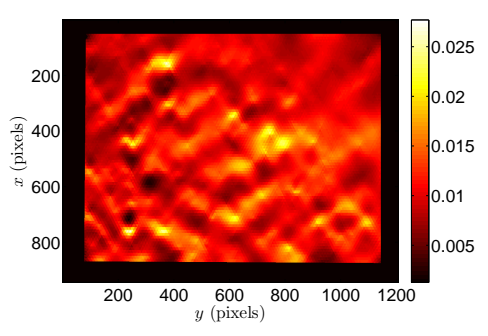

(c) Point 2, $\epsilon_{x x}$

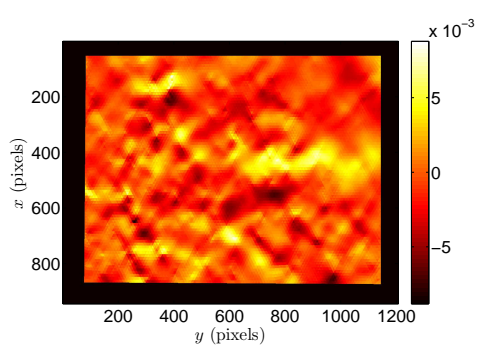

(e) Point $3, \epsilon_{x x}$

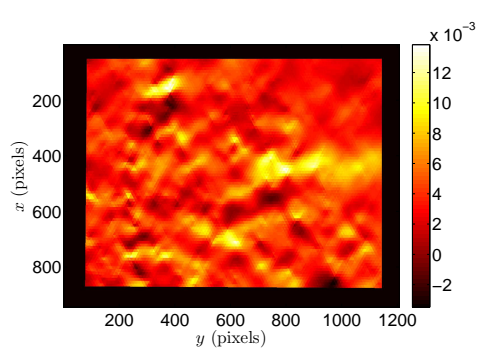

(g) Point $4, \epsilon_{x x}$

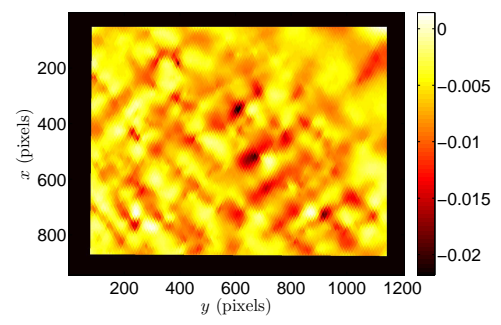

(b) Point 1, $\epsilon_{y y}$

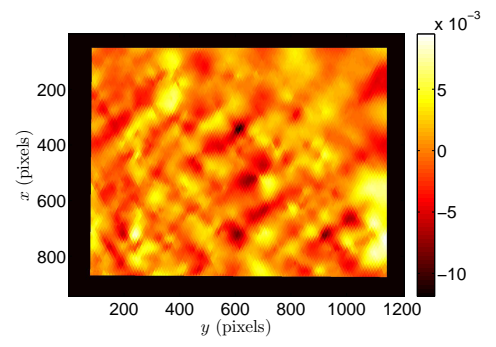

(d) Point 2, $\epsilon_{y y}$

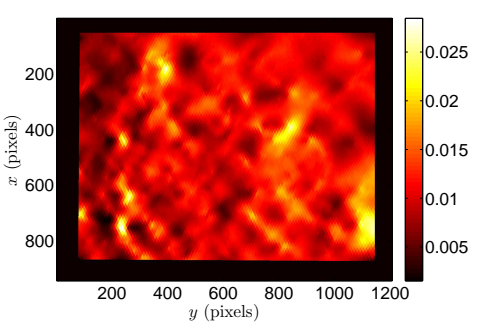

(f) Point 3, $\epsilon_{y y}$

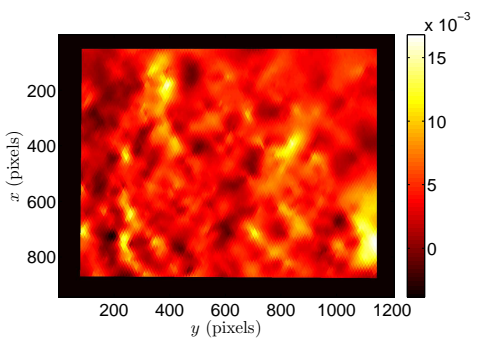

(h) Point 4, $\epsilon_{y y}$

Figure 22: Measured strain fields for first cycle at the mesoscale in the $x$ (left) and $y$ (right) directions of experiment SBE-1. (a) and (b) correspond to loading point 1 (see Figure 23), (c) and (d) correspond to loading point 2, (e) and (f) correspond to loading point $3,(\mathrm{~g})$ and $(\mathrm{h})$ correspond to loading point $4 . \quad 1$ pixel $\leftrightarrow 6.7 \mu \mathrm{m}$ 
From the first loading step strain bands are observed in both directions (Figure 22(a-b)). The detected strain bands coincide for the $\epsilon_{x x}$ and $\epsilon_{y y}$ maps. However, it is noted that $\epsilon_{x x}$ strains are positive in the bands while $\epsilon_{y y}$ are negative. Over the square loading path the size of these strain bands increases (Figure 22(c-f)). At the third point of the loading process new branches arise in $\epsilon_{y y}$ field over the evaluated zone. When the actuators in both directions are unloaded both strain maps (Figure 22(g-h)) have multiple bands. However, the dominant branch is observed in the central left part of the $\epsilon_{x x}$ strain field. From the correlation residuals it can be noted that the left part of the band is the source of the microcrack. As the strain band progresses to the right no damage (i.e., microcrack) is observed.

Figure 23 shows the strain response to a square loading for the first cycle. The strain components in the loading direction of the sample (Figure 23(a)) have a more complex response than observed for equibiaxial tests (see Figure 9(a)). When the first axis (i.e., 1-direction) is loaded or unloaded, the second one has a constant load level (see Section 2) and vice versa. The strain levels reach higher values (i.e., $1.5 \%$ ) than those obtained for the equibiaxial history (i.e., $0.32 \%$ ). The strain during the unloading phase of the second axis (i.e., unloading in the 2-direction) does not return to the reference point, which is interpreted as combined effects of plasticity and damage. 


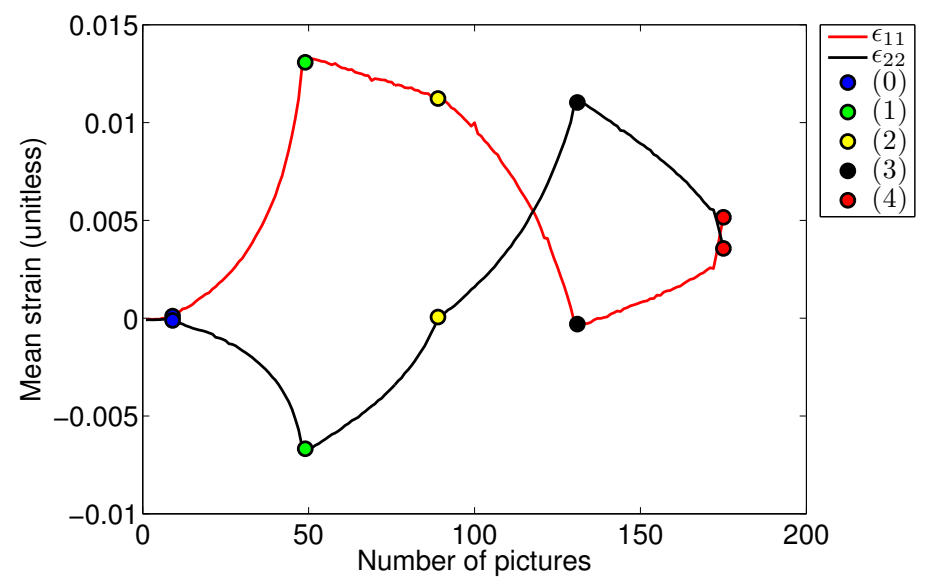

(a)

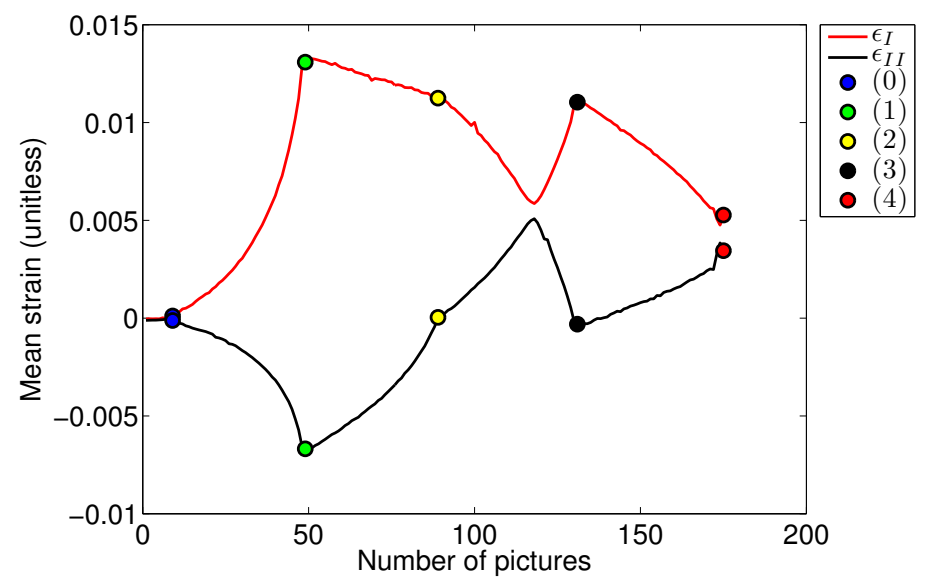

(b)

Figure 23: Strain histories describing the first cycle of experiment SBE-1 $\left(F_{\max }=40 \mathrm{kN}\right)$. (a) Axial strain in the two loading directions. (b) Mean eigen strains. Blue, green, yellow, black and red circles correspond to five characteristic points of square experiment (see Figure 5(b))

The SBE-1 test is designed so that it reaches the same load level as EBE1 , and hence it is expected that the crack network forms at the first loading 
cycle. Figure 24 shows the correlation and mechanical residuals obtained for the first cycle corresponding to point 2 (Figure 23(b)). The correlation residual map (Figure 24(a)) indicates that the gauge zone of the cross-shaped sample is much more affected by damage than EBE-1 test (Figure 10(a)) although the load level is similar. Mechanical residuals (Figure 10(b)) also show a higher level. Moreover, the equibiaxial test gave rise to an isotropic distribution of micro-cracks whereas the square loading induces very clearly preferential orientation of the micro-cracks along the two diagonal directions (colinear to vectors $(1, \pm 1)$ ). This property is expected to have further consequences on crack growth made easier, and crack coalescence rendered more difficult in perpendicular directions. The residual maps confirm the assumption that the square path is more damaging than an equibiaxial one.

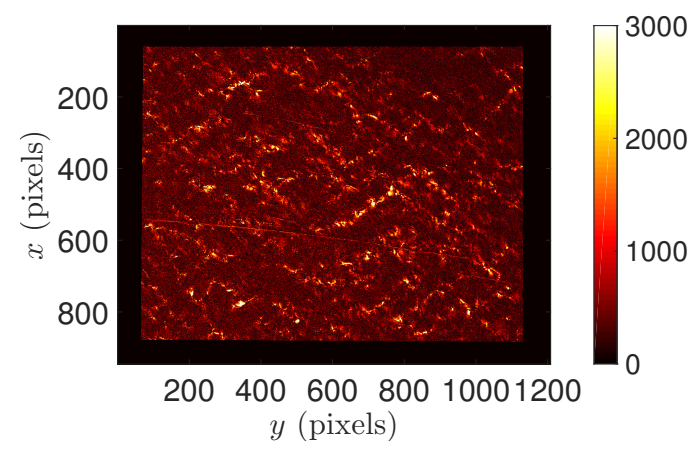

(a)

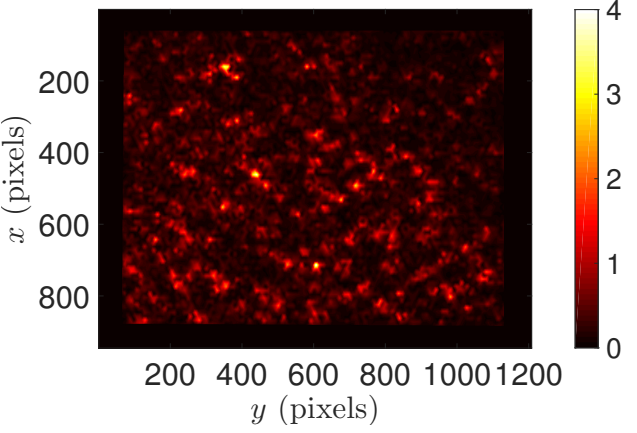

(b)

Figure 24: Mesoscale (a) correlation (in gray levels) and (b) mechanical residual maps corresponding to the maximum applied load level (i.e., $F_{1}=F_{2}=40 \mathrm{kN}$ or point 2) of the first cycle of experiment SBE-1. 1 pixel $\leftrightarrow 6.7 \mu \mathrm{m}$ 


\subsubsection{Strain history}

Figure 25 illustrates the strain histories of the first square experiment. From the presented mean axial strain histories the levels increase until cycle 100 at which stage the experiment is interrupted.

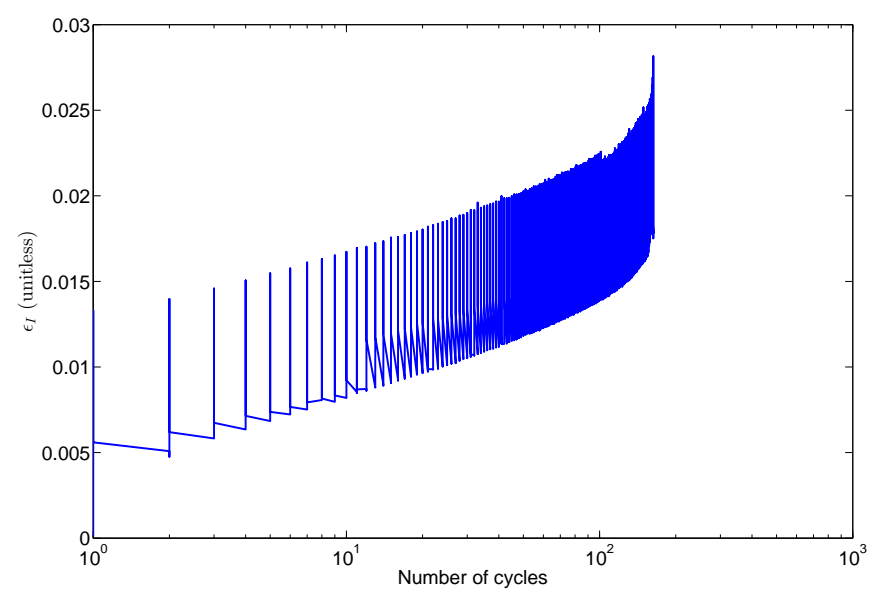

(a)

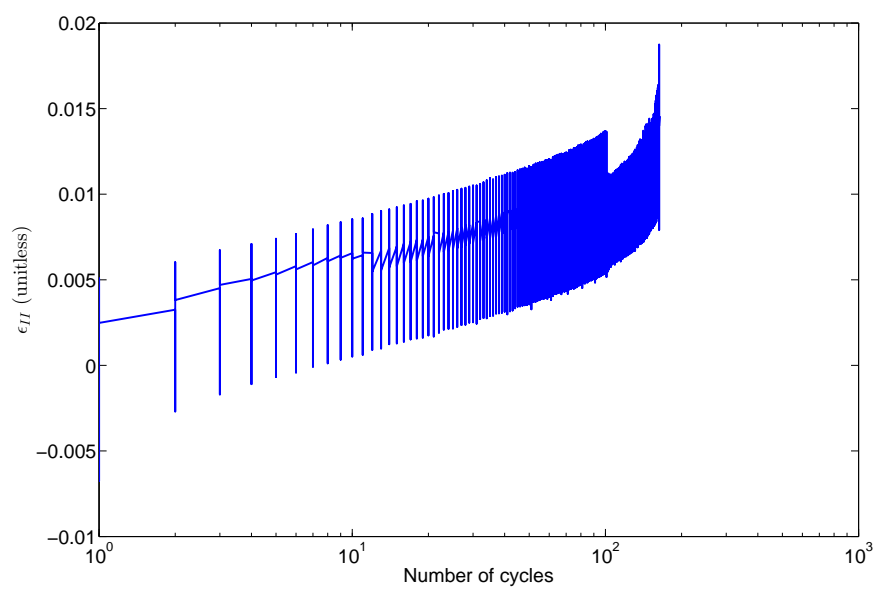

(b)

Figure 25: Measured (a) $\epsilon_{I}$ and (b) $\epsilon_{I I}$ history at mesoscale of experiment SBE-1. 
In order to see how the detected increase of strain levels reflects the material response, the strain amplitudes are determined from the peak values of the strain histories (i.e., minimum and maximum strain levels for a given cycle). Figure 26 shows the strain amplitudes evaluated at both scales. Differences between the macro- and mesoscales reach levels of $0.05 \%$, which corresponds to a flexure of the sample. However, strain amplitudes on both scales follow the same trend. After 10 cycles, the strain amplitudes start to increase until the experiment is stopped (after 100 cycles) due to data acquisition problems. This interruption caused the sudden drop of measured strains (Figure 25) and their amplitudes (Figure 26). The jump is more evident on the $\epsilon_{I I}$ and $\Delta \epsilon_{I I}$ data. The $\epsilon_{I}$ strain history (Figure 25(a)) does not report such a discontinuity, which leads to the conclusion that it did not accelerate the crack network growth. 


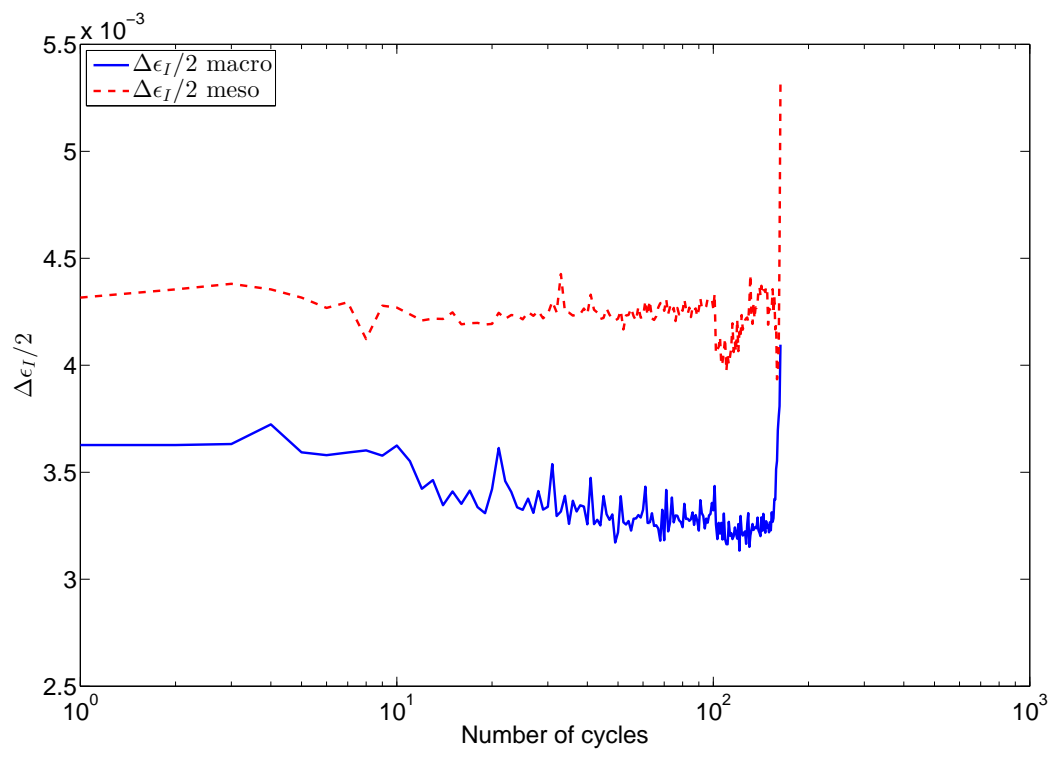

(a)

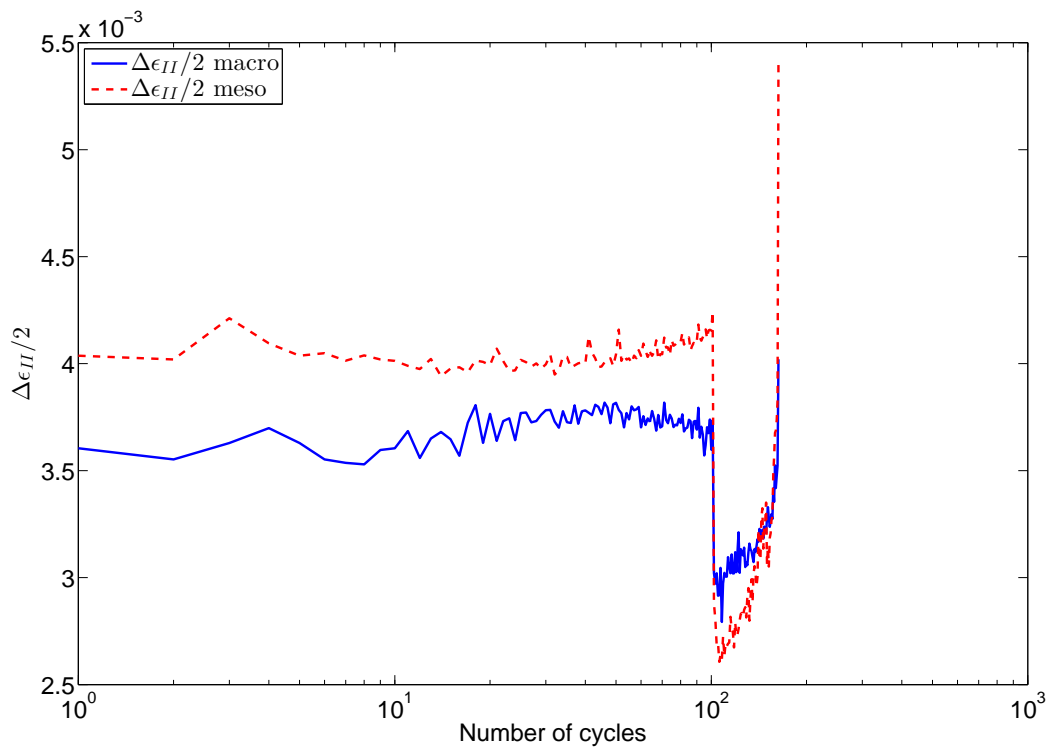

(b)

Figure 26: Measured strain amplitudes (a) $\epsilon_{I}$ and (b) $\epsilon_{I I}$ at macro- and mesoscales of experiment SBE-1 


\subsubsection{Crack network formation}

The normalized correlation and mechanical residuals exhibit less fluctuations than those observed on the strain data. The increase of residuals is easier to detect in order to determine the crack network growth. Figure 27(a) shows the change of $\hat{\Phi}_{c}$ and $\tilde{\Phi}_{m}$ with respect to the number of cycles. Many microcracks appear during the first cycle. Further cycles only give rise to a slow and progressive initiation of additional microcracks, which accelerate with the number of cycles up to failure. In Figure 27(b) correlation and mechanical residuals are shown as functions of the eigen strain amplitude measured on the mesoscale. Consistently with the previous observations, higher fluctuations of the strain data are observed while the residuals show higher sensitivity to crack network growth. 


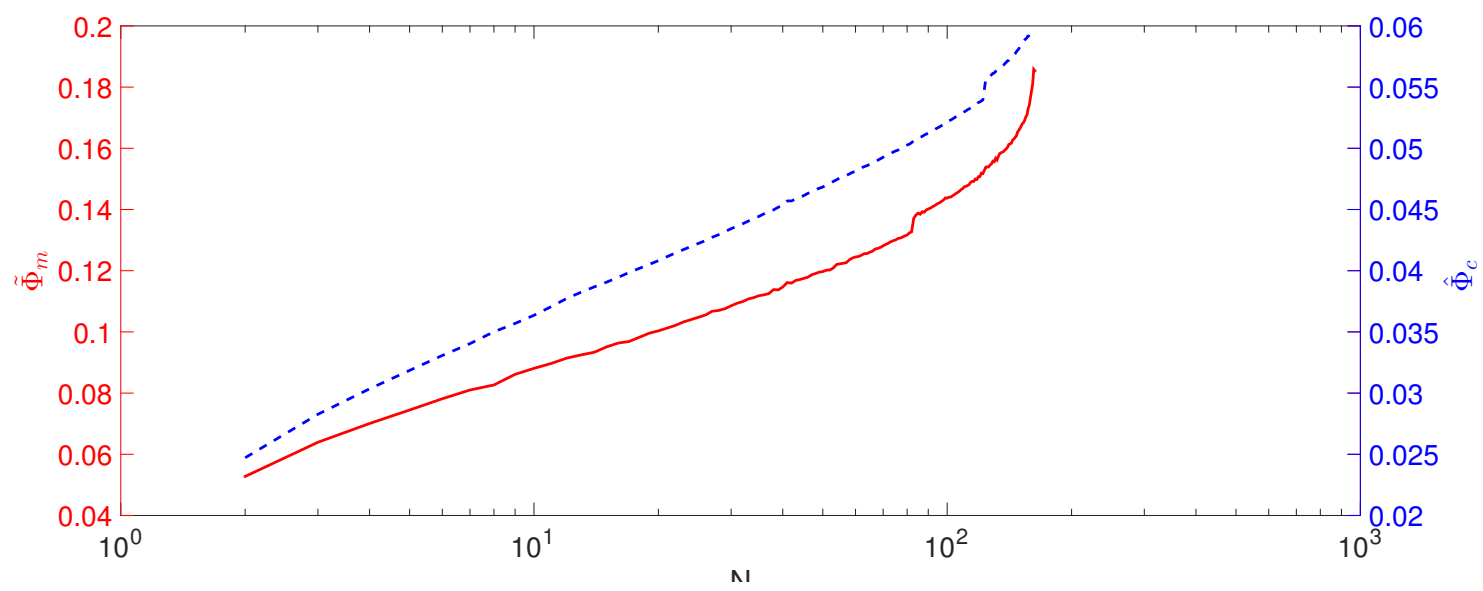

(a)

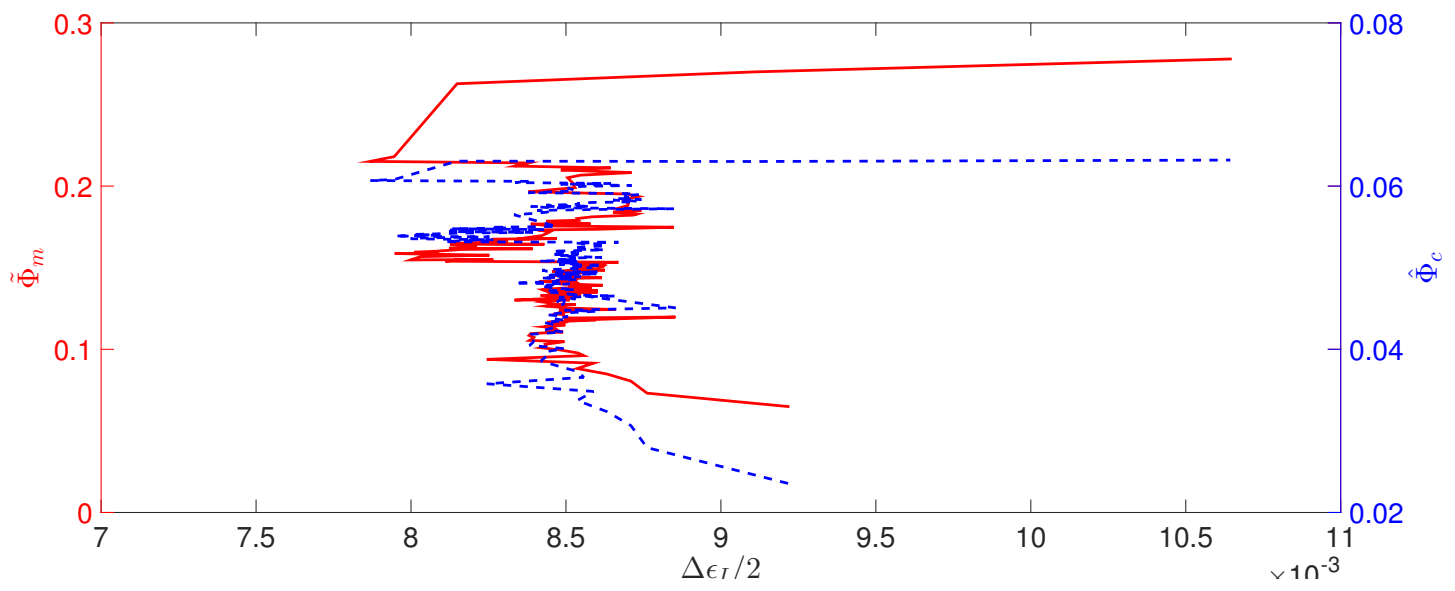

(b)

Figure 27: (a) Change of mechanical $\tilde{\Phi}_{m}$ and correlation residuals $\hat{\Phi}_{c}$ with the number of cycles of experiment SBE-1. (b) Change of $\tilde{\Phi}_{m}$ and $\hat{\Phi}_{c}$ with respect to the strain amplitude $\Delta \epsilon_{1} / 2$ at the mesoscale

Figure 28 shows the residual maps for different cycles. The crack network grows gradually as the number of cycles increases. Mechanical residuals first increase slowly, and progressively more quickly. In the middle of the 
figure at cycle 100 a macrocrack appears (Figure 28(c)) and subsequently (Figure 28(d)) this crack dominates. The mechanical residual maps show very similar features as the correlation residuals, albeit with a lower spatial resolution. 


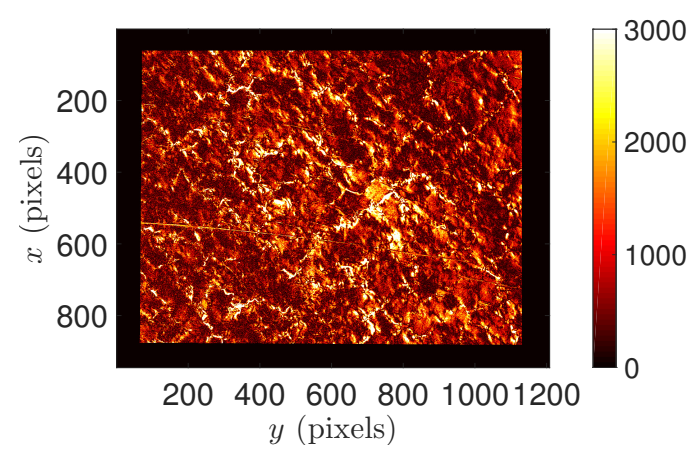

(a) Cycle 50

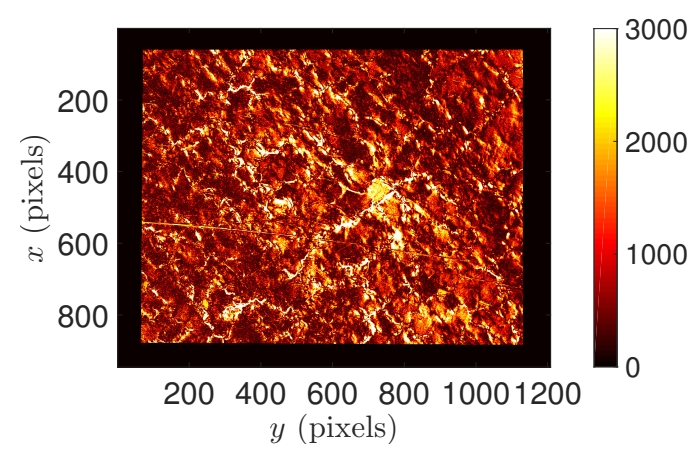

(c) Cycle 100

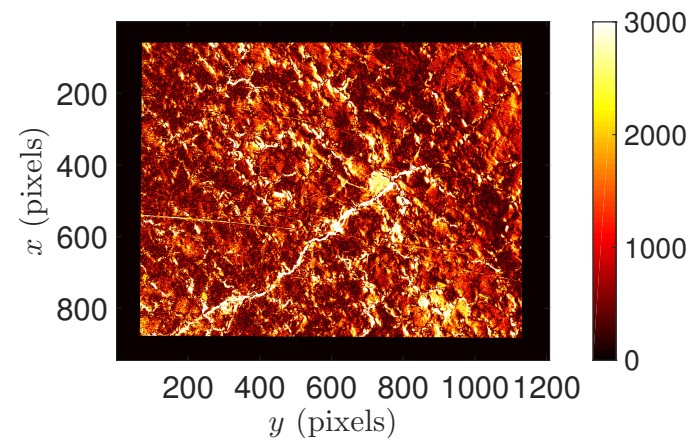

(e) Cycle 160

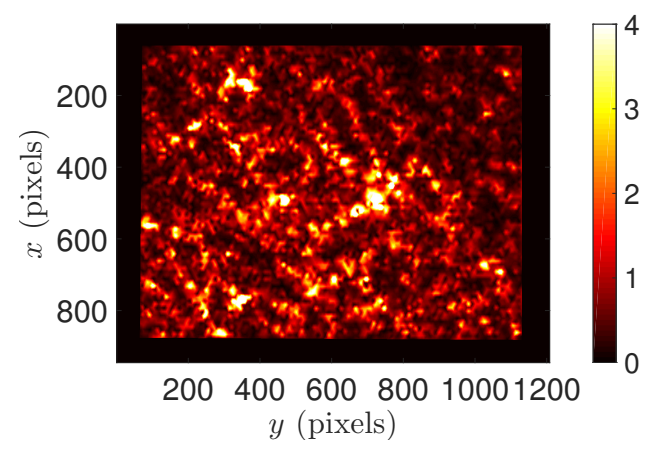

(b) Cycle 50

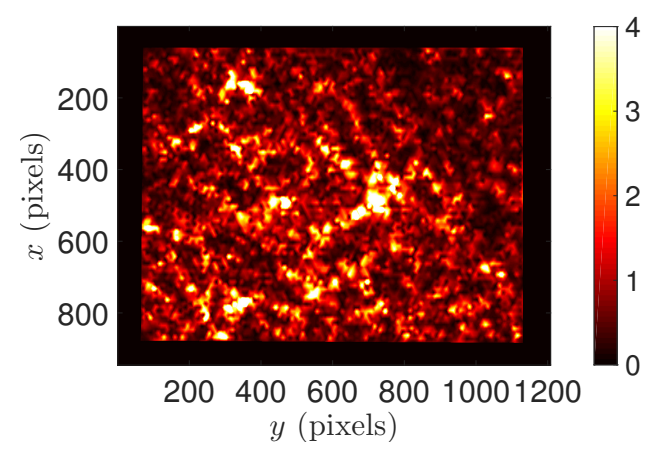

(d) Cycle 100

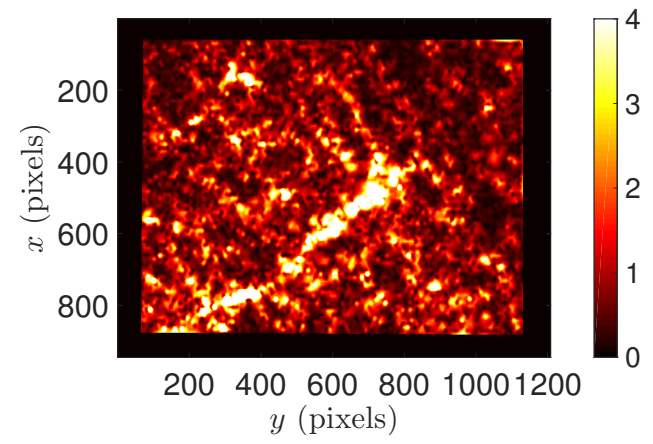

(f) Cycle 160

Figure 28: Mesoscale (gray level) correlation residual (a,c,e) and mechanical residual (b,d,f) maps corresponding to the maximum applied load level for different numbers of cycles of experiment SBE-1. 1 pixel $\leftrightarrow 6.7 \mu \mathrm{m}$ 


\subsection{Microcrack initiation in SBE-2 test}

The initiation of microcracks will be investigated for the SBE-2 experiment where the maximum load equals $20 \mathrm{kN}$ (i.e., half that of SBE-1, but equal to that of EBE-2). Figure 29 shows the mean axial strains during the first cycle. The first two steps are consistent with a linear elastic behavior. However, when the first axis is unloaded (Figure 29, point (2) to (3)) a nonlinear response occurs. This is due to microdamage caused by debonding [33]. The maximum value $\epsilon_{I}=0.4 \%$ is reached at the third loading point. Hence, the strain amplitude of the maximum eigen strain is defined as the difference between the third and fourth loading points of the square loading path (see Figure 5(b)).

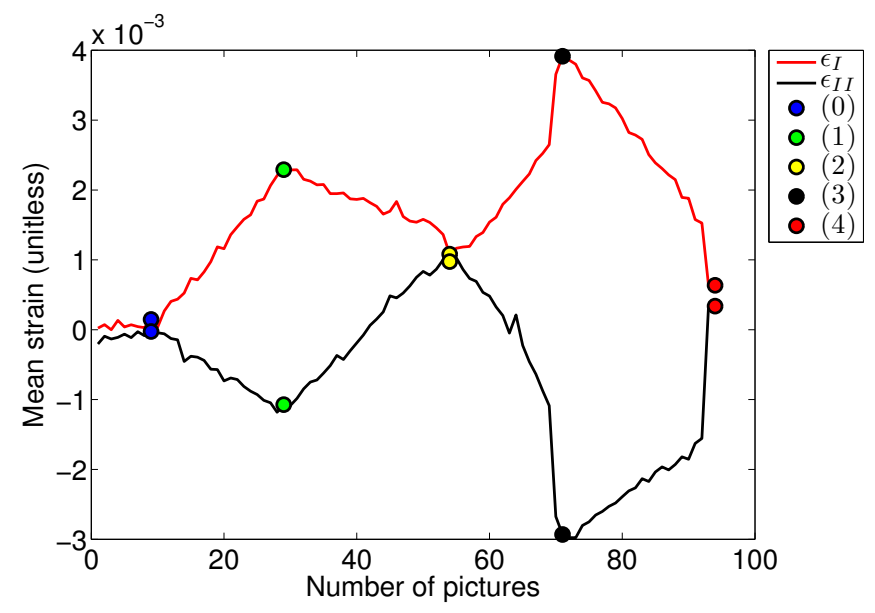

(a)

Figure 29: Mean eigen strains for the first cycle of SBE-2 test $\left(F_{\max }=20 \mathrm{kN}\right)$ at the mesoscale. Five characteristic points (Figure 5(b)) of the square experiment are depicted

Figure 30 shows the residual maps obtained for the maximum level of 
$\epsilon_{I}$. In the correlation residuals no discontinuity pops out even though the strain history revealed a nonlinear response. During loading the strain fields are uniform while at the mesoscale strain localization is observed. However, in the unloaded point uniform fields are reported. Mechanical residuals (Figure 30(b)) display some spatial fluctuations but it is not possible to distinguish meaningful features from noise in the observed zone. Since neither the map of correlation residual nor that of the mechanical residuals reveal any discontinuities, it can be concluded that the crack network has not been initiated yet at the first cycle.

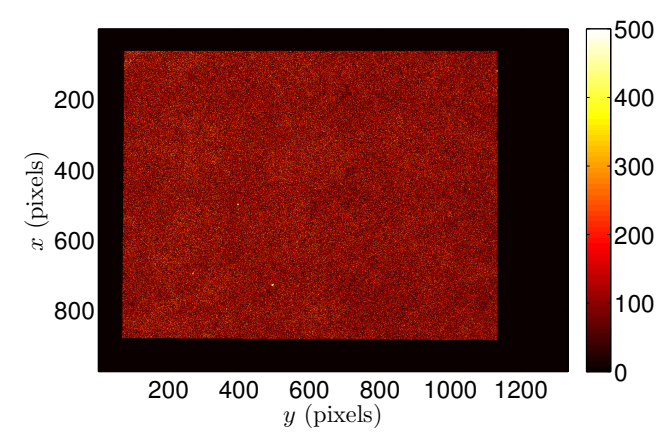

(a)

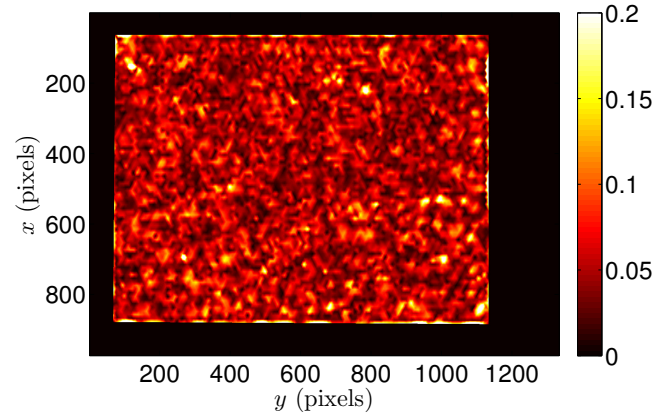

(b)

Figure 30: Mesoscale (a) correlation (in gray levels) and (b) mechanical residual maps corresponding to the load level when the maximum value of $\epsilon_{I}$ is reached (i.e., $F_{1}=$ $0, F_{2}=20 \mathrm{kN}$, or point 3 ) for the first cycle of SBE-2 test. 1 pixel $\leftrightarrow 6.7 \mu \mathrm{m}$

As the fatigue test proceeds, the correlation and mechanical residuals are studied (Figure 31(a)). A very good correspondence is noted with no significant changes until cycle 60,000. Figure 31(b) shows the change of normalized correlation and mechanical residuals as functions of the maximum strain am- 
plitude. The two residuals follow the same trend. At the beginning of the test very small fluctuations are observed. Conversely, when microcracks initiate (i.e., after cycle 50,000 as shown below) a rapid increase of strain amplitude is noted.

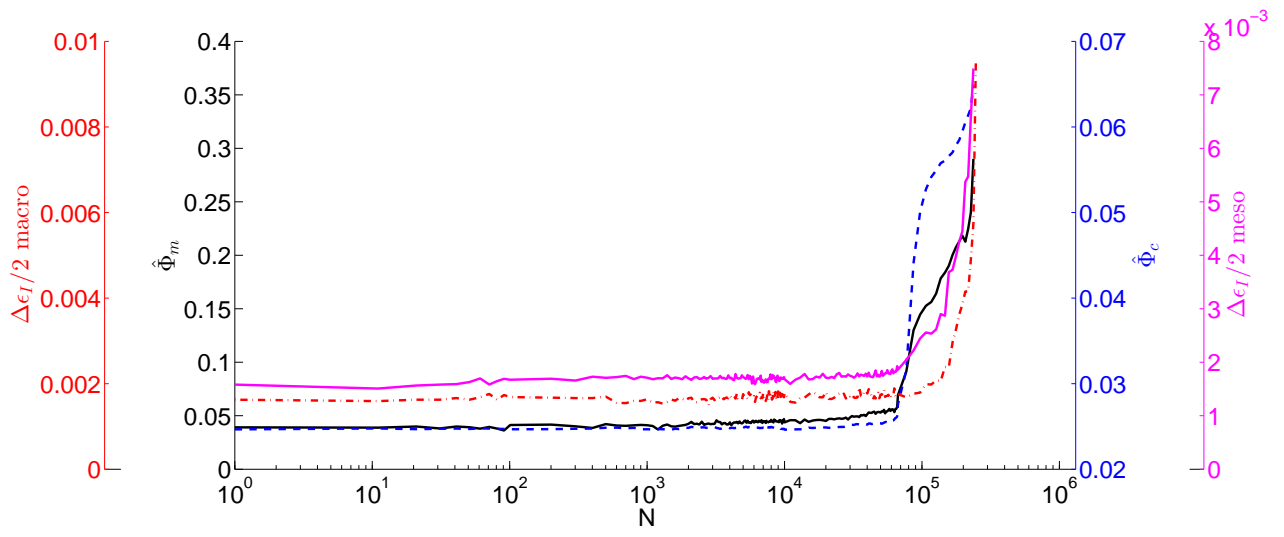

(a)

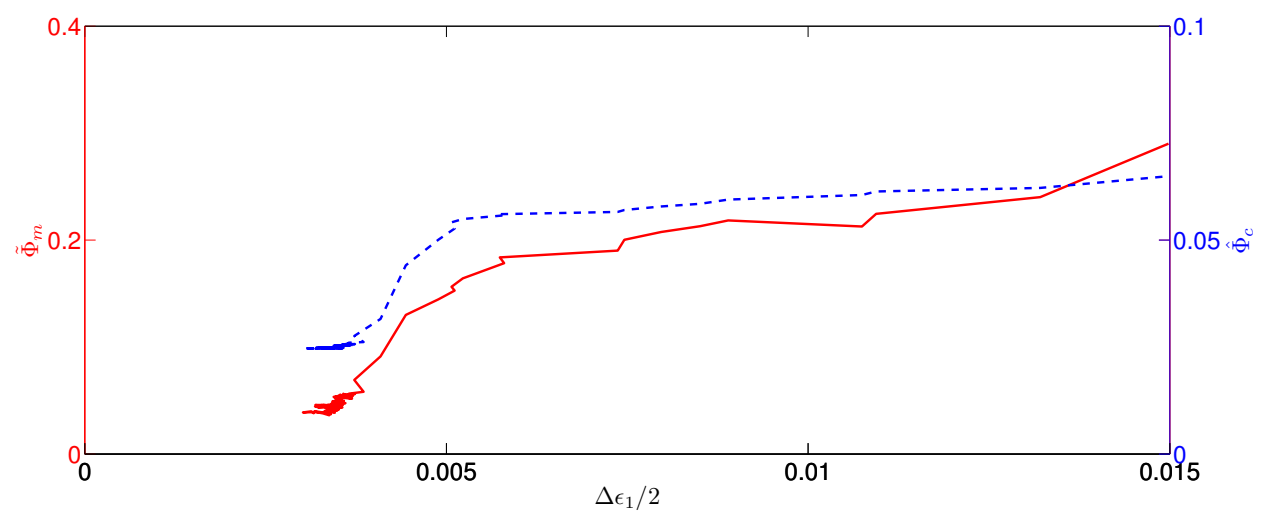

(b)

Figure 31: (a) Changes of mesoscale mechanical $\tilde{\Phi}_{m}$ and normalized correlation residuals $\hat{\Phi}_{c}$ with the number of cycles of experiment SBE-2. For comparison purposes $\Delta \epsilon_{I}$ are reported at macro- and mesoscales. (b) Changes of $\tilde{\Phi}_{m}$ and $\hat{\Phi}_{c}$ with respect to the strain amplitude $\Delta \epsilon_{I}$ at the mesoscale. 1 pixel $\leftrightarrow 6.7 \mu \mathrm{m}$ 
The corresponding correlation residual maps are illustrated in Figure 32. The first detected discontinuity (Figure 32(e)) corresponds approximately to cycle 50,000. The maximum eigen strain amplitude on the mesoscale follows the same trend as the residuals. The macroscopic strain amplitude also increases less since microcracks are not captured as easily as on the mesoscale. Comparing the residual maps obtained at cycle 100,000 (Figure 32(f)) with the previous ones shows that one dominant macrocrack has formed. Since the crack surface was sliding between 50,000 and 100,000 cycles metal dust started to appear on the surface of the sample, which increases the correlation and the mechanical residuals. This effect leads the residuals to increase in the center of the observed ROI since the conservation of gray levels is violated. However it did not prevent convergence of DIC outside this zone, and hence the results are partly trustworthy. As the number of cycles increases more dust is produced (Figure $32(\mathrm{~g})$ and $(\mathrm{h})$ ) and hence the quantitative value of the global residuals cannot be considered as reliable, although its rapid increase remains a very precise indication of macrocrack initiation and propagation. 


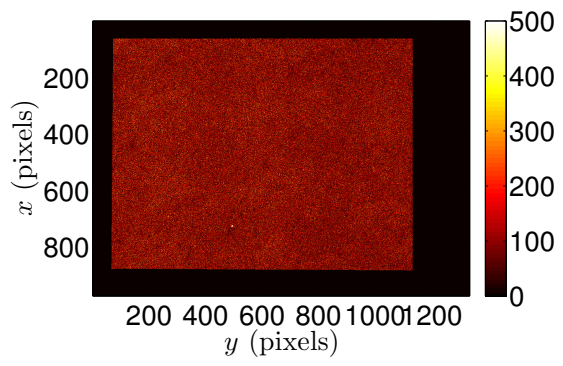

(a) Cycle 10

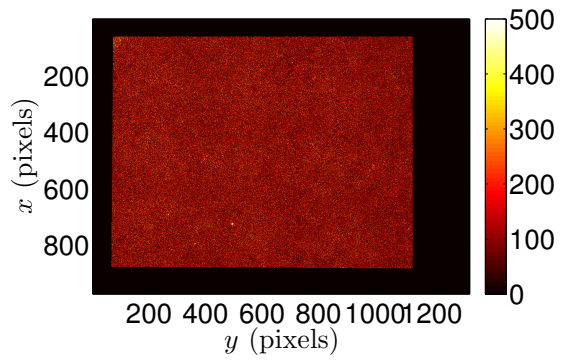

(c) Cycle 1,000

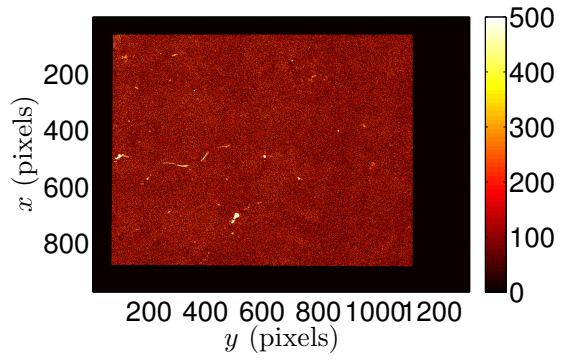

(e) Cycle 50,000

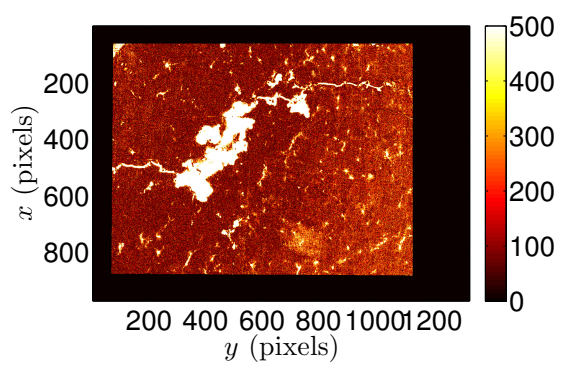

(g) Cycle 150,000

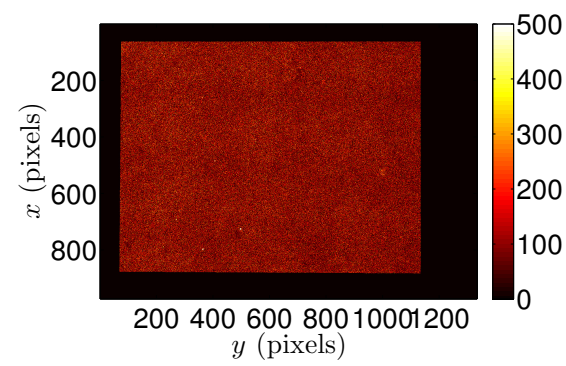

(b) Cycle 100

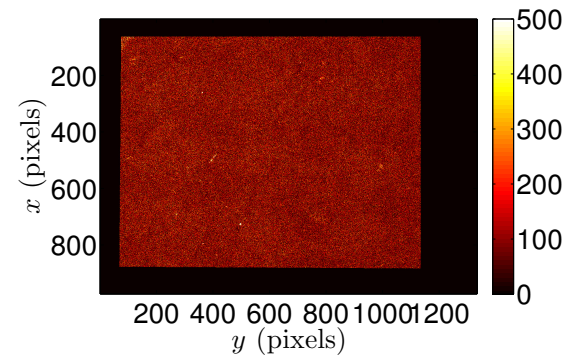

(d) Cycle 10,000

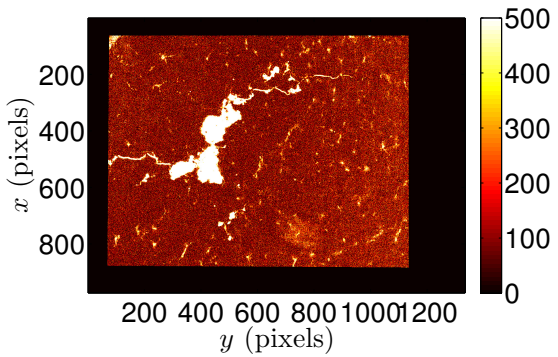

(f) Cycle 100,000

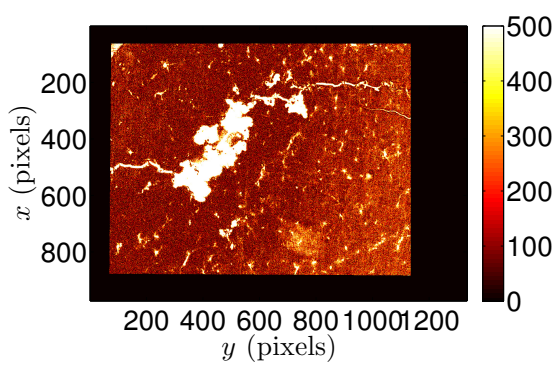

(h) Cycle 220,000

Figure 32: Correlation residual maps (in gray levels) for different numbers of cycles at mesoscale of experiment SBE-2. 1 pixel $\stackrel{57}{\leftrightarrow} 6.7 \mu \mathrm{m}$ 


\section{Conclusion}

The objective of the present experimental study was to study the biaxial fatigue behavior of SG cast iron under multiaxial loading conditions. Uniaxial and biaxial experiments $[33,32]$ revealed that the material was quasibrittle. Both sides of the cross-shaped samples were observed at macro- and mesoscales. Images captured at the macroscale were dealing with an artificial pattern, while the images at the mesolevel used the natural texture of cast iron. The latter was poor and very difficult to handle with classical DIC algorithms (i.e., local and global approaches). The lack of gray level contrast was successfully surpassed with regularized DIC [31].

Two different loading histories were applied in order to study the material behavior under equibiaxial and square loading regimes. The latter one is seldom studied. First, strain histories were extracted from the measured displacement fields. The results observed on the two scales showed an overall good agreement although images were acquired on two opposite sides of the sample. When detected the systematic difference in mean strain is interpreted as flexure. The origin of such a flexural mode is not known, it may be present from the beginning of the test because of imperfect alignments, or induced by a dissymmetry in surface damage. In order to study the flexural mechanism the strain histories have been extracted and compared on macroand mesoscales. Biaxial experiments EBE-1 and SBE-1 show differences between the strain paths during the first cycle. Conversely, when $20-\mathrm{kN}$ load levels are prescribed (i.e., EBE-2 and SBE-2) a very good agreement between 
the strain histories is noted. Measured strain components in the loading directions (i.e., $\epsilon_{11}$ and $\epsilon_{22}$ ) coincide from the beginning of the experiment. As the crack network establishes strain paths evaluated on the two scales deviate. It is concluded that experiments without crack initiation at the first cycle introduce flexural effects when the strain amplitude starts to increase (see Appendix C). Ignoring it would lead to biased conclusions on microcrack onset and fatigue development. Mesoscale results revealed microcrack network initiation and growth as the sample is cycled. The two applied loading paths yield completely different strain histories. An equibiaxial loading regime leads to the same strain history for the components measured in the loading direction and the eigen strains. Conversely, square histories are more complex and more damaging.

Four biaxial fatigue tests (i.e., two equibiaxial and two square loadings) have been performed up to $1.5 \times 10^{6}$ cycles. In those cases the crack network formation either started at the very first loading cycle or after some numbers of cycles. The evaluation of the mean strain data from DIC could not be directly used to assess the crack network formation and growth with the number of cycles, as the softening expected from the initiation of microcracks was masked by strain uncertainties during most of the fatigue test. The mean strains calculated over a large ROI could not detect the discontinuities very early on as microcracks are initially quite small. However, correlation and mechanical residuals were shown to be very useful to monitor the crack network initiation and growth. For both global residuals, the change of resid- 
uals is not fluctuating as much as the strain signals. Moreover, correlation residual maps reveal extremely powerful and accurate to locate microcracks at pixel spatial resolution. Crack growth could be monitored on mesoscale observations.

The goal of two series of biaxial experiments was to investigate the influence of the applied loading path on the fatigue life time. From the analyzed results it is concluded that the more complex biaxial (i.e., square) loading path is more damaging than the proportional loading history. Hence, a lower life time is to be expected from more complex loading histories (Table 2). This type of test is a good candidate for assessing the predictive power of various models accounting for microcrack initiation, propagation, and coa-

lescence. Having access to the kinematic fields at two different scales will enable for the analysis of multiscale constitutive postulates or assumptions.

\section{Acknowledgments}

This work was supported by a grant from Région Île-de-France (plateforme francilienne d'expérimentation mécanique de troisième génération). ZT thanks Campus France that supported his stay at LMT through an Eiffel scholarship. 


\section{Appendix A. A priori displacement analysis for mesoscale images}

This first analysis on the natural pattern aims to evaluate the quality of measured fields when the solution is known. Hence, the picture of Figure 6(b) is artificially deformed. A sine wave is considered [4], see Figure A.33. A linear interpolation of the gray levels is used to generate the picture in the deformed configuration. The parametric analysis is performed via RT3-DIC for different initial regularization lengths $\ell_{m}$ and the relaxation process for which in every new initialized computation $\ell_{m}$ was divided by 2 [31]. Even though the texture is poor 10-pixel elements are chosen since regularization is considered.

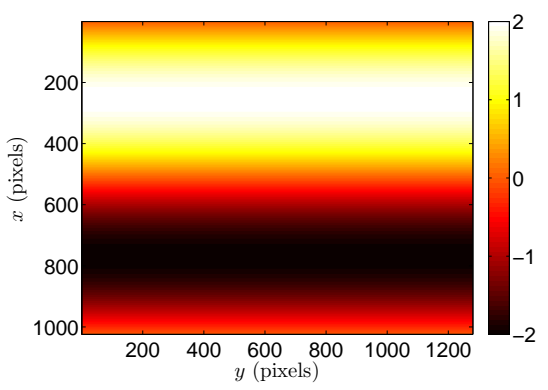

(a)

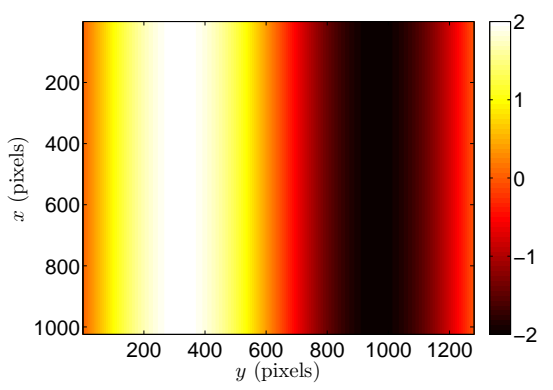

(b)

Figure A.33: Prescribed displacement field in the sine wave test case in (a) vertical and (b) horizontal directions. The displacements are expressed in pixels. 1 pixel $\leftrightarrow 6.7 \mu \mathrm{m}$

Figure A.34 shows the standard displacement error comparing the prescribed (i.e., known) and measured displacement fields. It can be noted that higher regularization lengths $\left(1024<\ell_{m}<256\right.$ pixels $)$ yield higher displacement errors since small displacement fluctuations are smeared out. The 
lowest standard displacement error is recorded when a regularization length of 128 pixels is considered while for smaller $\ell_{m}$ lengths (i.e., 32 and 64 pixels) the error is higher (but still not as high as for $1024<\ell_{m}<256$ pixels). In the end of the relaxation process the computations with initial regularization lengths of 1024, 64 and 32 pixels yield approximately the same standard displacement error. When comparing these results with the analysis performed on an artificial texture [31] and a prescribed displacement field the same trend is observed for the error changes during the relaxation process although with differing values at the end.

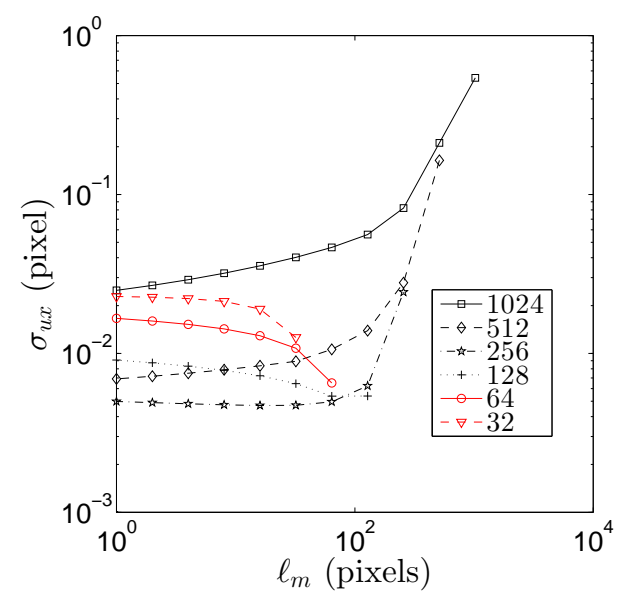

(a)

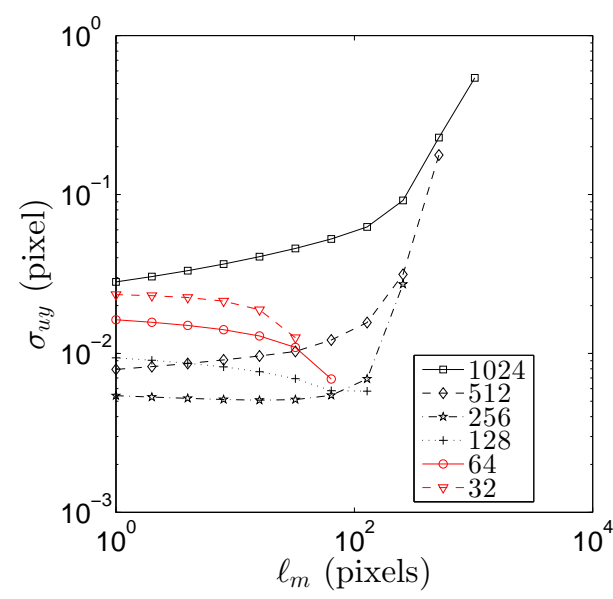

(b)

Figure A.34: Standard displacement errors in (a) the vertical and (b) horizontal directions for different regularization lengths $\ell_{m}$ when $\ell_{b} / \ell_{m}=1 / 2$. The legend indicates the initial regularization length $\ell_{m}$ (in pixels). 1 pixel $\leftrightarrow 6.7 \mu \mathrm{m}$

The change of dimensionless correlation residuals $\hat{\Phi}_{c}$ (Figure A.35(a)) leads to higher values for $\ell_{m}=1024$ and 512 pixels. For the other regular- 
ization lengths the correlation residuals reach similar levels (i.e., the results are trustworthy). This property is even noted during the relaxation process. When compared to the artificial texture [31] higher lengths $\ell_{m}$ do not affect the residuals as strongly, which leads to the conclusion that it is necessary to put more effort to successfully perform RT3-DIC on poor textures. For larger regularization lengths, the measured field displays less equilibrium gap. This is explained by the increase of its weight in the total functional. The most favorable zone in the present case lies in the range of initial values $\left(128<\ell_{m}<512\right.$ pixels) for which the equilibrium gap is independent of the initial value of $\ell_{m}$.

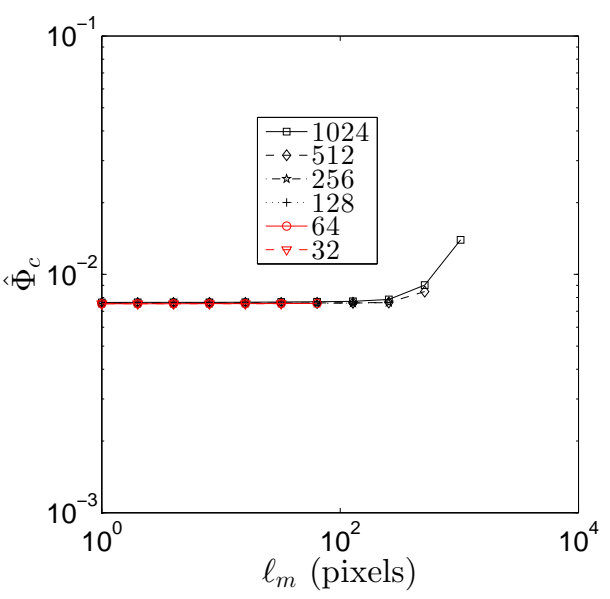

(a)

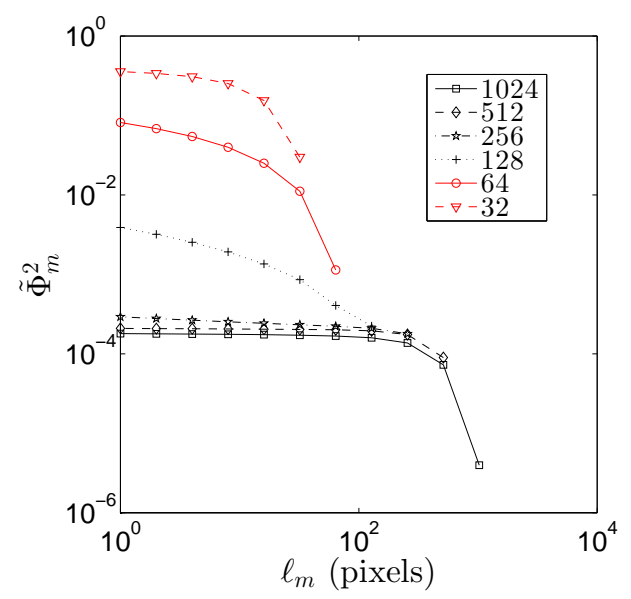

(b)

Figure A.35: Change of (a) the dimensionless correlation residuals and (b) equilibrium residuals for different regularization lengths and different initializations. Note the narrow range of variation of $\hat{\Phi}_{c} . \quad 1$ pixel $\leftrightarrow 6.7 \mu \mathrm{m}$ 


\section{Appendix B. Displacement and strain resolution assessment}

To assess the standard displacement and strain resolutions nine images are captured when the specimen is mounted and the load level is constant (approximately equal to $0 \mathrm{~N}$ ). From the measured displacement field $u_{i}^{\text {meas }_{j}}$ for image $i$ in the axis $j$ and corresponding strain level $\epsilon_{i}^{\text {meas }_{j}}$ the displacement and strain resolutions are given by

$$
\gamma_{u}^{j}=\sqrt{\frac{1}{\tilde{n} n_{D O F}} \sum_{i=1}^{\tilde{n}}\left(u_{i}^{\text {meas }_{j}}-\bar{u}_{i}^{\text {meas }_{j}}\right)^{2}}
$$

and

$$
\gamma_{\epsilon}^{j}=\sqrt{\frac{1}{\tilde{n} n_{e l}} \sum_{i=1}^{\tilde{n}}\left(\epsilon_{i}^{\text {meas }_{j}}-\bar{\epsilon}_{i}^{\text {meas }_{j}}\right)^{2}}
$$

where $\boldsymbol{}$ denotes the mean of the vector collecting all the nodal displacements or the average strain field, $\tilde{n}$ the number of images, $n_{D O F}$ the number of kinematic unknowns, and $n_{e l}$ the number of elements. The level of $\gamma_{u}$ and $\gamma_{\epsilon}$ for the studied experiments is given in Table B.3. 
Table B.3: Standard displacement and strain resolutions at macro- and mesoscales in $x$ and $y$-directions

\begin{tabular}{|c|c|c|c|c|c|}
\hline & Scale & $\begin{array}{c}\gamma_{u}^{x} \\
(\text { pixel) }\end{array}$ & $\begin{array}{c}\gamma_{u}^{y} \\
\text { (pixel) }\end{array}$ & $\gamma_{\epsilon}^{x}$ & $\gamma_{\epsilon}^{y}$ \\
\hline \multirow{2}{*}{ EBE-1 } & Macro $\sharp$ & 0.015 & 0.012 & $4 \times 10^{-4}$ & $4 \times 10^{-4}$ \\
\hline & meso ${ }^{b}$ & 0.010 & 0.012 & $2 \times 10^{-4}$ & $2 \times 10^{-4}$ \\
\hline \multirow{2}{*}{ EBE-2 } & Macro $\sharp$ & 0.010 & 0.011 & $3 \times 10^{-4}$ & $3 \times 10^{-4}$ \\
\hline & $\operatorname{meso}^{b}$ & 0.013 & 0.013 & $2 \times 10^{-4}$ & $2 \times 10^{-4}$ \\
\hline \multirow{2}{*}{ SBE-1 } & Macro $\sharp$ & 0.013 & 0.015 & $3 \times 10^{-4}$ & $4 \times 10^{-4}$ \\
\hline & $\operatorname{meso}^{b}$ & 0.021 & 0.027 & $4 \times 10^{-4}$ & $4 \times 10^{-4}$ \\
\hline \multirow{2}{*}{ SBE-2 } & Macro $\sharp$ & 0.014 & 0.012 & $3 \times 10^{-4}$ & $3 \times 10^{-4}$ \\
\hline & meso ${ }^{b}$ & 0.020 & 0.018 & $5 \times 10^{-4}$ & $5 \times 10^{-4}$ \\
\hline
\end{tabular}




\section{Appendix C. Onset of flexure vs. microcrack initiation}

When analyzing the proposed biaxial experiments differences between the strain amplitudes on macro- and mesoscales were noted. The assumption was made that flexure occurred. In order to evaluate the origin of such an effect the strain histories are extracted over the whole image series (see Figures C.36 and C.37). The four experiments investigated herein have two flexural modes.

First, for EBE-1 test flexure was noted from the first cycle on. The material response expressed in term of strain histories $\epsilon_{11}$ (see Figure 36(a)) and $\epsilon_{22}$ (see Figure 36(b)) estimated on the two scales results in different strain amplitudes. The mean fluctuations measured via DIC gauge show a very good agreement for the maximum $\epsilon_{11}$ strain levels. This is not the case when the sample is unloaded. At this stage a gap equal to $0.2 \times 10^{-3}$ is reported between the macro- and mesoscale (Figure C.36). Conversely, the $\epsilon_{22}$ histories measured on the two scales reach the same strain levels while a deviation is noted for the maximum load levels. Therefore, it is believed that the discrepancy is due to imperfect alignments since the strain difference is established from the beginning of the experiment between the two sides of the cross-shaped sample. 


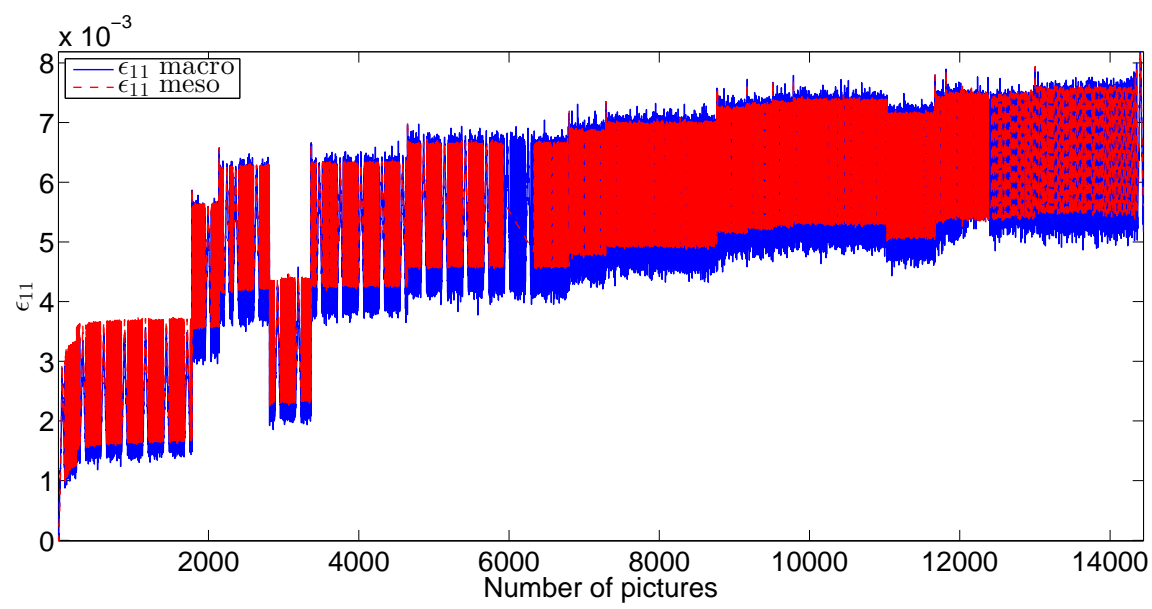

(a)

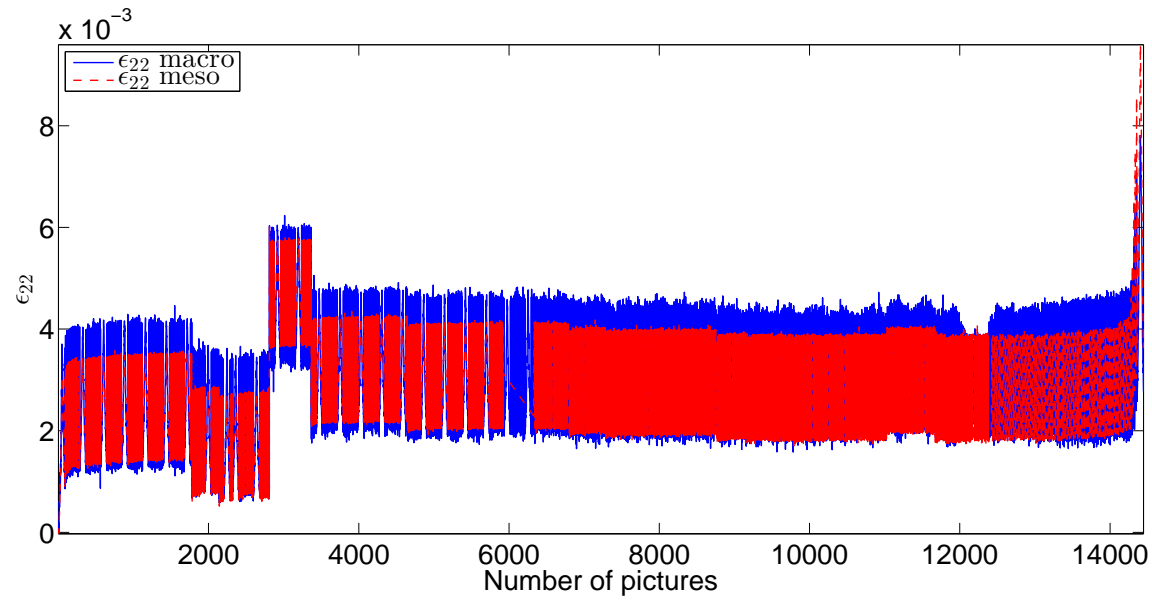

(b)

Figure C.36: Measured (a) $\epsilon_{11}$ and (b) $\epsilon_{22}$ history at macro- and mesoscales for EBE-1

The second flexural mode is visible on SBE-2 experiment (Figure C.37). The strain histories (i.e., $\epsilon_{11}$ and $\epsilon_{22}$ ) extracted on both scales coincide. However, after the 1,800th picture the measured mean strain levels in the gauge zone start to differ. This phenomenon is believed to be due to be induced by 
a dissymmetry in surface damage.

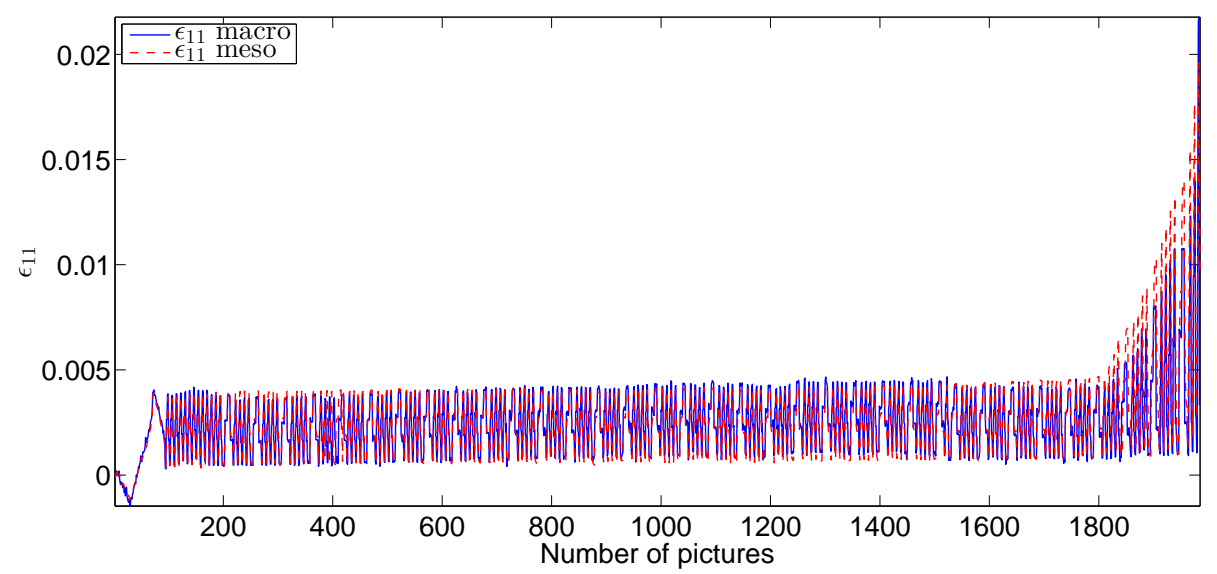

(a)

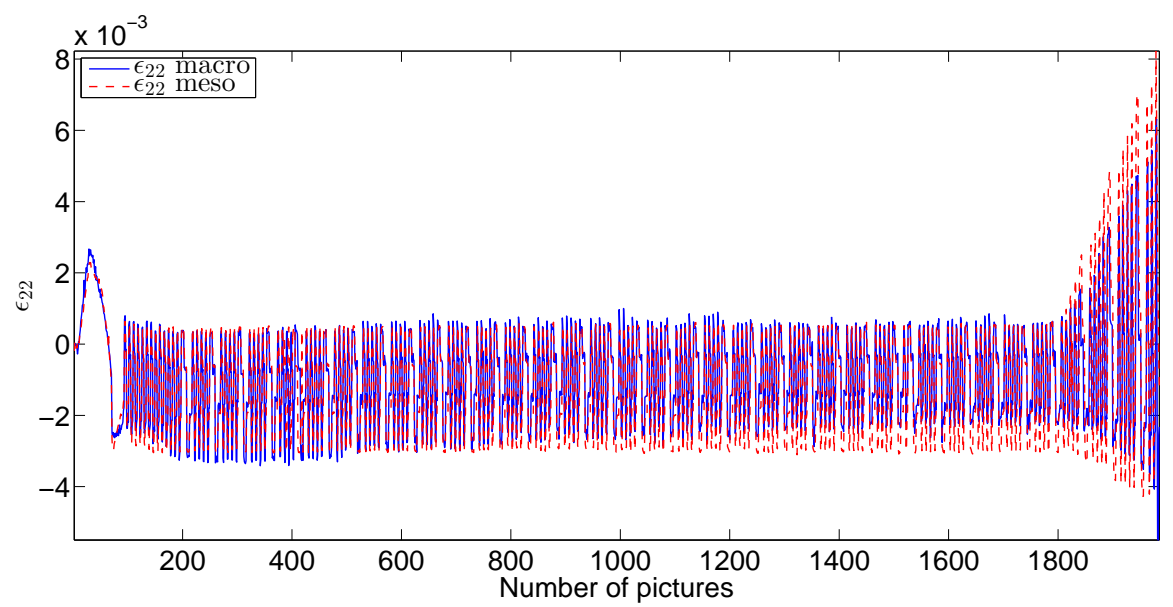

(b)

Figure C.37: Measured (a) $\epsilon_{11}$ and (b) $\epsilon_{22}$ history at macro- and mesoscales for SBE-2

Flexural modes for the in-plane biaxial experiments analyzed in this work are summarized in Table C.4. 
Table C.4: Flexural modes from $\epsilon_{11}$ and $\epsilon_{22}$ strain histories

\begin{tabular}{lccc} 
Test case & Strain direction & Flexure & Crack initiation \\
\hline EBE-1 & $\epsilon_{11}$ & Yes & No \\
& $\epsilon_{22}$ & Yes & No \\
\hline EBE-2 & $\epsilon_{11}$ & No & Yes \\
& $\epsilon_{22}$ & No & Yes \\
SBE-1 & $\epsilon_{11}$ & No & Yes \\
& $\epsilon_{22}$ & Yes & No \\
SBE-2 & $\epsilon_{11}$ & No & Yes \\
& $\epsilon_{22}$ & No & Yes \\
\hline
\end{tabular}




\section{Appendix D. List of acronyms}

CCD charge coupled device

CNRS Centre National de la Recherche Scientifique

DIC digital image correlation

EBE equibiaxial experiment

ENS Ecole Normale Supérieure

LMT Laboratory of Mechanics and Technology

ROI region of interest

R-DIC regularized digital image correlation

RT3-DIC regularized digital image correlation with T3 discretizations

SBE $\quad$ square biaxial experiment

SG spheroidal graphite

T3 3-noded triangular element 


\section{References}

[1] J. Abanto-Bueno and J. Lambros. Experimental determination of cohesive failure properties of a photodegradable copolymer. Exp. Mech., 45(2):144-152, 2005.

[2] A. Bataille and T. Magnin. Surface damage accumulation in low-cycle fatigue: Physical analysis and numerical modeling. Acta Metall. Mater., 42(11):3817-3825, 1994.

[3] G. Besnard, F. Hild, and S. Roux. "Finite-element" displacement fields analysis from digital images: Application to Portevin-Le Chatelier bands. Exp. Mech., 46:789-803, 2006.

[4] M. Bornert, F. Brémand, P. Doumalin, J.C. Dupré, M. Fazzini, M. Grédiac, F. Hild, S. Mistou, J. Molimard, J.J. Orteu, L. Robert, Y. Surrel, P. Vacher, and B. Wattrisse. Assessment of digital image correlation measurement errors: Methodology and results. Exp. Mech., 49(3):353$370,2009$.

[5] G.B. Broggiato. Adaptive image correlation technique for full-field strain measurement. In C. Pappalettere, editor, 12th Int. Conf. Exp. Mech., pages 420-421. McGraw Hill, Lilan (Italy), 2004.

[6] M.C. Casperson, J.D. Carroll, J. Lambros, H. Sehitoglu, and R.H. Dodds Jr. Investigation of thermal effects on fatigue crack closure using multiscale digital image correlation experiments. Int. J. Fat., 61:10-20, 2014. 
[7] L. Chevalier, S. Calloch, F. Hild, and Y. Marco. Digital image correlation used to analyze the multiaxial behavior of rubber-like materials. Eur. J. Mech. A/Solids, 20:169-187, 2001.

[8] D. Claire, F. Hild, and S. Roux. A finite element formulation to identify damage fields: The equilibrium gap method. Int. J. Num. Meth. Engng., 61(2):189-208, 2004.

[9] P.F.P. de Matos and D. Nowell. Experimental and numerical investigation of thickness effects in plasticity-induced fatigue crack closure. Int. J. Fat., 31:1795-1804, 2009.

[10] P.Y. Decreuse, S. Pommier, M. Poncelet, and B. Raka. A novel approach to model mixed mode plasticity at crack tip and crack growth. Experimental validations using velocity fields from digital image correlation. Int. J. Fat., 42:271-283, 2012.

[11] C. Doudard, M. Poncelet, S. Calloch, C. Boué, F. Hild, and A. Galtier. Determination of an hcf criterion by thermal measurements under biaxial cyclic loading. Int. J. Fat., 29:748-757, 2007.

[12] A. El Bartali, V. Aubin, and S. Degallaix. Fatigue damage analysis in a duplex stainless steel by digital image correlation technique. Fat. Fract. Eng. Mat. Struct., 31(2):137-151, 2008.

[13] B. Fedelich. A stochastic theory for the problem of multiple surface crack coalescence. Int. J. Fract., 91:23-45, 1998. 
[14] F. Frémy, S. Pommier, M. Poncelet, B. Raka, E. Galenne, S. Courtin, and J.-C. Le Roux. Load path effect on fatigue crack propagation in I + II + III mixed mode conditions - Part 1: Experimental investigations. Int. J. Fat., 62:104-112, 2014.

[15] R. Hamam, F. Hild, and S. Roux. Stress intensity factor gauging by digital image correlation: Application in cyclic fatigue. Strain, 43:181192, 2007.

[16] F. Hild and S. Roux. Evaluating Damage with Digital Image Correlation: A. Introductory Remarks and Detection of Physical Damage. In G.Z. Voyiadjis, editor, Handbook of Damage Mechanics, pages 1255-1275, New York, NY (USA), 2015. Springer.

[17] T. Hoshide and D.F. Socie. Crack nucleation and growth modeling in biaxial fatigue. Eng. Fract. Mech., 29(3):287-299, 1988.

[18] J. Lambros J.D. Carroll, W. Abuzaid and H. Sehitoglu. High resolution digital image correlation measurements of strain accumulation in fatigue crack growth. Int. J. Fat., 57:140-150, 2013.

[19] A.M. Korsunsky, X. Song, J. Belnoue, T. Jun, F. Hofmann, P.F.P. De Matos, D. Nowell, D. Dini, O. Aparicio-Blanco, and M.J. Walsh. Crack tip deformation fields and fatigue crack growth rates in Ti-6Al-4Va. Int. J. Fat., 31(11-12):1771-1779, 2009. 
[20] U. Lindborg. A statistical model for the linking of microcracks. Acta Metall., 17(4):521-526, 1969.

[21] N. Malésys, L. Vincent, and F. Hild. A probabilistic model to predict the formation and propagation of crack networks in thermal fatigue. Int. J. Fat., 31(3):565-574, 2009.

[22] F. Mathieu, F. Hild, and S. Roux. Identification of a crack propagation law by digital image correlation. Int. J. Fat., 36:146-154, 2012.

[23] Y. Ochi, A. Ishii, and S.K. Sasaki. An experimental and statistical investigation of surface fatigue crack initiation and growth. Fat. Fract. Eng. Mat. Struct., 8:327-339, 1985.

[24] M. Poncelet, G. Barbier, B. Raka, S. Courtin, R. Desmorat, J.C. LeRoux, and L. Vincent. Biaxial High Cycle Fatigue of a type 304L stainless steel: Cyclic strains and crack initiation detection by digital image correlation. Eur. J. Mech. A/Solids, 29:810-825, 2010.

[25] J. Rupil, S. Roux, F. Hild, and L. Vincent. Fatigue microcrack detection with digital image correlation. J. Strain Analysis, 46(6):492-509, 2011.

[26] J. Rupil, L. Vincent, F. Hild, and S. Roux. Probabilistic modeling of mesocrack initiations in 3041 stainless steel. Int. J. Multiscale Comput. Eng., 9(4):445-458, 2011.

[27] J.P. Sermage, J. Lemaitre, and R. Desmorat. Multiaxial creep-fatigue 
under anisothermal conditions. Fat. Fract. Eng. Mat. Struct., 23(3):241$252,2000$.

[28] Y. Sun, J. Pang, C. Wong, and F. Su. Finite-element formulation for a digital image correlation method. Appl. Optics, 44(34):7357-7363, 2005.

[29] M.A. Sutton. Computer vision-based, noncontacting deformation measurements in mechanics: A generational transformation. Appl. Mech. Rev., 65(AMR-13-1009):050802, 2013.

[30] M.A. Sutton, W. Zhao, S.R. McNeill, J.D. Helm, R.S. Piascik, and W.T. Riddel. Local crack closure measurements: Development of a measurement system using computer vision and a far-field microscope, pages 145-156. ASTM, 1999.

[31] Z. Tomičević, F. Hild, and S. Roux. Mechanics-aided digital image correlation. J. Strain Analysis, 48:330-343, 2013.

[32] Z. Tomičević, J. Kodvanj, and F. Hild. Characterization of the nonlinear behavior of nodular graphite cast iron via inverse identification. Analysis of biaxial tests. Europ. J. Mech. A/Solids, 59:195-209, 2016.

[33] Z. Tomičević, J. Kodvanj, and F. Hild. Characterization of the nonlinear behavior of nodular graphite cast iron via inverse identification. Analysis of uniaxial tests. Europ. J. Mech. A/Solids, 59:140-154, 2016.

[34] S. Vanlanduit, J. Vanherzeele, R. Longo, and P. Guillaume. A digital 
image correlation method for fatigue test experiments. Optics Lasers Eng., 47:371-378, 2009.

[35] F. Yusof, P. Lopez-Crespo, and P.J. Withers. Effect of overload on crack closure in thick and thin specimens via digital image correlation. Int. J. Fat., 56:17-24, 2013.

[36] T. Zhai, A.J. Wilkinson, and J.W. Martin. A crystallographic mechanism for fatigue crack propagation through grain boundaries. Acta Mat., 48(20):4917-4927, 2000. 


\section{List of Figures}

1 Metallography of the studied SG cast iron . . . . . . . 5

2 Maltese cross-shaped specimen designed for in-plane biaxial

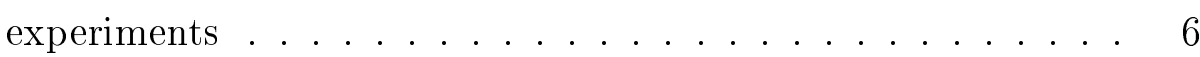

3 Two-scale experimental setup. (a) Mesoscale optical setup, (b) macroscale optical setup, (c) CCD camera Dalsa ${ }^{\mathrm{TM}}$ with telecentric lens ( $\times 4$ magnification), (d) macroscale image, (e) beam-splitter, (f) CCD camera PixelFly ${ }^{\mathrm{TM}}$ with telecentric lens ( $\times 1$ magnification), (g) DedoCool ${ }^{\mathrm{TM}}$ light source, (h) mesoscale image . . . . . . . . . . . . . . . 8

4 The two prepared sides of the cross-shaped specimen for twoscale observations. (a) Artificial texture for macro- and (b) polished surface for mesoscale observations . . . . . . . . 9

$5 \quad$ One cycle for (a) equibiaxial and (b) square loading histories. Equibiaxial loading consists of two (i.e., (1)-(2)) characteristic points while square history has four (i.e., (1)-(2)-(3)-(4)) . . . 10

6 DIC gauges at the two scales. (a) Macroscale and (b) mesoscale DIC gauges depicted as square (blue) zones over the unstructured T3 mesh with 10 pixel elements (red). All dimensions are expressed in pixels . . . . . . . . . . . . . 16 
7 Measured strain fields for first cycle at the macroscale in the $x$ (left) and $y$ (right) directions of experiment EBE-1. (a) and (b) correspond to loading point 1 (see Figure 5(a)), (c) and (d) correspond to loading point 2. The arrows indicate the strained bands. 1 pixel $\leftrightarrow 48 \mu \mathrm{m} \ldots \ldots . . . . . . . .18$

8 Measured strain fields for the first cycle at the mesoscale in the $x$ (left) and $y$ (right) directions of experiment EBE-1. (a) and (b) correspond to loading point 1 (see Figure 5(a)), (c) and (d) correspond to loading point 2. 1 pixel $\leftrightarrow 6.7 \mu \mathrm{m} .20$

9 Strain histories of the first cycle of EBE-1 experiment $\left(F_{\max }=\right.$ $40 \mathrm{kN}$ ). (a) Mean strains $\epsilon_{11}$ and $\epsilon_{22}$ in the loading directions of the sample. (b) Mean eigen strains. Blue, green and yellow circles correspond to the three characteristic points of equibiaxial experiments (see Figure 5(a)) . . . . . . . . 21

10 Mesoscale (a) correlation (in gray level) and (b) mechanical residual maps corresponding to maximum load level (i.e., $F_{1}=$ $F_{2}=40 \mathrm{kN}$ ) for the first cycle of EBE-1 experiment. The arrows indicate the detected cracks. 1 pixel $\leftrightarrow 6.7 \mu \mathrm{m} \ldots 22$

11 Measured (a) $\epsilon_{I}$ and (b) $\epsilon_{I I}$ histories with respect to the number of cycles of experiment EBE-1. . . . . . . . . 24

12 Measured amplitudes for (a) $\epsilon_{I}$ and (b) $\epsilon_{I I}$ at macro- and mesoscales of experiment EBE-1 ........... 25 
13 (a) Change of mesoscale mechanical $\tilde{\Phi}_{m}$ and normalized correlation residuals $\hat{\Phi}_{c}$ with the number of cycles of experiment EBE-1. (b) Corresponding change of $\tilde{\Phi}_{m}$ and $\hat{\Phi}_{c}$ with respect to the strain amplitude $\Delta \epsilon_{1} / 2 \ldots \ldots \ldots \ldots$

14 Mesoscale (gray level) correlation (left) and mechanical (right) residual maps corresponding to the maximum applied load level for different number of cycles of experiment EBE-1.

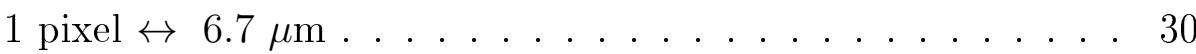

15 Measured strain fields corresponding to the maximum applied load level at the mesoscale in the $x$ (left) and $y$ (right) directions for different numbers of cycles of experiment EBE-1. 1 pixel $\leftrightarrow 6.7 \mu \mathrm{m} \ldots \ldots \ldots \ldots \ldots . \ldots . \ldots . \ldots$

16 Mean eigen strains for the first cycle of equibiaxial experiment EBE-2 (i.e., $F_{\max }=20 \mathrm{kN}$ ) at the mesoscale. Blue, green and yellow circles correspond to the three characteristic points of Figure $5(\mathrm{a}) \ldots \ldots \ldots \ldots \ldots \ldots \ldots$

17 Mesoscale (a) correlation (in gray levels) and (b) mechanical residual maps corresponding to a maximum applied load $\left(F_{1}=F_{2}=20 \mathrm{kN}\right)$ for the first cycle of experiment EBE-2.

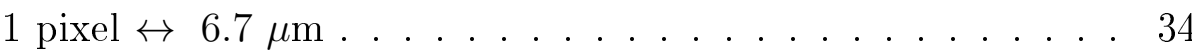


18 Correlation between mechanical $\left(\tilde{\Phi}_{m}\right)$ and normalized correlation $\left(\hat{\Phi}_{c}\right)$ residuals from whole series of images acquired in experiment EBE-2 at mesoscale. The red circle denotes the point where the applied load level has been increased . . . . . 35

19 (a) Changes of mesoscale mechanical $\tilde{\Phi}_{m}$ and normalized correlation residuals $\hat{\Phi}_{c}$ with the number of cycles of experiment EBE-2. For comparison purposes $\Delta \epsilon_{I}$ are reported at macroand mesoscales. The vertical dashed line shows when the applied load has been increased. (b) Changes of $\tilde{\Phi}_{m}$ and $\hat{\Phi}_{c}$ with the strain amplitude $\Delta \epsilon_{I}$ at the mesoscale $\ldots \ldots \ldots 36$

20 Mesoscale (gray level) correlation residual maps for different numbers of cycles of experiment EBE-2. 1 pixel $\leftrightarrow 6.7 \mu \mathrm{m} .38$

21 Measured strain fields for first cycle at the macroscale in the $x$ (left) and $y$ (right) directions of experiment SBE-1. (a) and (b) correspond to loading point 1 (see Figure 5(b)), (c) and (d) to loading point 2, (e) and (f) to loading point 3, (g) and (h) to loading point $4 . \quad 1$ pixel $\leftrightarrow 48 \mu \mathrm{m} \quad \ldots \ldots \ldots$. . . 40

22 Measured strain fields for first cycle at the mesoscale in the $x$ (left) and $y$ (right) directions of experiment SBE-1. (a) and (b) correspond to loading point 1 (see Figure 23), (c) and (d) correspond to loading point 2, (e) and (f) correspond to loading point $3,(\mathrm{~g})$ and $(\mathrm{h})$ correspond to loading point 4 . 1 pixel $\leftrightarrow 6.7 \mu \mathrm{m} \ldots \ldots \ldots \ldots \ldots \ldots \ldots$ 
23 Strain histories describing the first cycle of experiment SBE-1 $\left(F_{\max }=40 \mathrm{kN}\right)$. (a) Axial strain in the two loading directions. (b) Mean eigen strains. Blue, green, yellow, black and red circles correspond to five characteristic points of square experiment (see Figure 5(b)) . . . . . . . . . . . . 44

24 Mesoscale (a) correlation (in gray levels) and (b) mechanical residual maps corresponding to the maximum applied load level (i.e., $F_{1}=F_{2}=40 \mathrm{kN}$ or point 2 ) of the first cycle of experiment SBE-1. 1 pixel $\leftrightarrow 6.7 \mu \mathrm{m} \ldots . . . . . .45$

25 Measured (a) $\epsilon_{I}$ and (b) $\epsilon_{I I}$ history at mesoscale of experiment

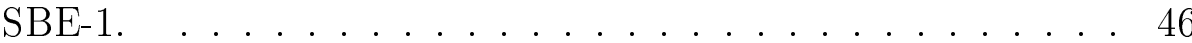

26 Measured strain amplitudes (a) $\epsilon_{I}$ and (b) $\epsilon_{I I}$ at macro- and mesoscales of experiment SBE-1 . . . . . . . . . 48

27 (a) Change of mechanical $\tilde{\Phi}_{m}$ and correlation residuals $\hat{\Phi}_{c}$ with the number of cycles of experiment SBE-1. (b) Change of $\tilde{\Phi}_{m}$ and $\hat{\Phi}_{c}$ with respect to the strain amplitude $\Delta \epsilon_{1} / 2$ at the mesoscale .................... 50

28 Mesoscale (gray level) correlation residual (a,c,e) and mechanical residual $(b, d, f)$ maps corresponding to the maximum applied load level for different numbers of cycles of experiment SBE-1. 1 pixel $\leftrightarrow 6.7 \mu \mathrm{m} \ldots \ldots . \ldots . \ldots 52$ 
29 Mean eigen strains for the first cycle of SBE- 2 test $\left(F_{\max }=20\right.$ $\mathrm{kN}$ ) at the mesoscale. Five characteristic points (Figure 5(b)) of the square experiment are depicted . . . . . . . . 53

30 Mesoscale (a) correlation (in gray levels) and (b) mechanical residual maps corresponding to the load level when the maximum value of $\epsilon_{I}$ is reached (i.e., $F_{1}=0, F_{2}=20 \mathrm{kN}$, or point 3) for the first cycle of SBE-2 test. 1 pixel $\leftrightarrow 6.7 \mu \mathrm{m} \ldots . .54$

31 (a) Changes of mesoscale mechanical $\tilde{\Phi}_{m}$ and normalized correlation residuals $\hat{\Phi}_{c}$ with the number of cycles of experiment SBE-2. For comparison purposes $\Delta \epsilon_{I}$ are reported at macroand mesoscales. (b) Changes of $\tilde{\Phi}_{m}$ and $\hat{\Phi}_{c}$ with respect to the strain amplitude $\Delta \epsilon_{I}$ at the mesoscale. 1 pixel $\leftrightarrow 6.7 \mu \mathrm{m} \quad 55$

32 Correlation residual maps (in gray levels) for different numbers of cycles at mesoscale of experiment SBE-2. 1 pixel $\leftrightarrow 6.7 \mu \mathrm{m} 57$

A.33 Prescribed displacement field in the sine wave test case in (a) vertical and (b) horizontal directions. The displacements are expressed in pixels. 1 pixel $\leftrightarrow 6.7 \mu \mathrm{m} \ldots . . . . . .661$

A.34 Standard displacement errors in (a) the vertical and (b) horizontal directions for different regularization lengths $\ell_{m}$ when $\ell_{b} / \ell_{m}=1 / 2$. The legend indicates the initial regularization length $\ell_{m}$ (in pixels). 1 pixel $\leftrightarrow 6.7 \mu \mathrm{m} \ldots \ldots . \ldots 62$ 
A.35 Change of (a) the dimensionless correlation residuals and (b) equilibrium residuals for different regularization lengths and different initializations. Note the narrow range of variation of $\hat{\Phi}_{c} .1$ pixel $\leftrightarrow 6.7 \mu \mathrm{m} \ldots \ldots . . \ldots . . . . . . . .663$

C.36 Measured (a) $\epsilon_{11}$ and (b) $\epsilon_{22}$ history at macro- and mesoscales for $\mathrm{EBE}-1 \ldots \ldots \ldots \ldots \ldots 7 . \ldots . \ldots . \ldots 67$

C.37 Measured (a) $\epsilon_{11}$ and (b) $\epsilon_{22}$ history at macro- and mesoscales for SBE-2 . . . . . . . . . . . . . . 68 


\section{List of Tables}

1 Chemical composition in vol. \% of the studied grade of SG cast iron ........................ 4

2 Definition of the equibiaxial and square loading paths, respectively referred to with the acronyms EBE and SBE ..... 11

B.3 Standard displacement and strain resolutions at macro- and mesoscales in $x$ - and $y$-directions $\ldots \ldots \ldots 65$

C.4 Flexural modes from $\epsilon_{11}$ and $\epsilon_{22}$ strain histories . . . . . . 69 